\title{
RANKL-mediated harmonious dialogue between fetus and mother guarantees smooth gestation by inducing decidual M2 macrophage polarization
}

\author{
Yu-Han Meng ${ }^{1,5}$, Wen-Jie Zhou ${ }^{1,2,3,5}$, Li-Ping Jin ${ }^{4}$, Li-Bing Liu ${ }^{1}$, Kai-Kai Chang ${ }^{1,2}$, Jie Mei ${ }^{1}$, Hui Li ${ }^{4}$, Jian Wang ${ }^{2}$, Da-Jin Lił ${ }^{* 1,2}$ and \\ Ming-Qing Li $\mathrm{i}^{*, 1,2,3}$
}

Decidual macrophages $(\mathrm{dM} \varphi$ ) contribute to maternal-fetal tolerance. However, the mechanism of $\mathrm{dM} \varphi$ differentiation during pregnancy is still largely unknown. Here, we report that receptor activator for nuclear factor- $\kappa$ B ligand (RANKL), secreted by human embryonic trophoblasts and maternal decidual stromal cells (DSCs), polarizes $\mathrm{dM} \varphi$ toward a M2 phenotype. This polarization is mediated through activation of Akt/signal transducer and activator of transcription 6 (STAT6) signaling, which is associated with the upregulation of histone H3 lysine-27 demethylase Jmjd3 and IRF4 in dM $\varphi$. Such differentiated dM $\varphi$ can induce a Th2 bias that promotes maternal-fetal tolerance. Impaired expression of RANKL leads to dysfunction of dM $\varphi$ in vivo and increased rates of fetal loss in mice. Transfer of RANK ${ }^{+} M \varphi$ reverses mouse fetal loss induced by M $\varphi$ depletion. Compared with normal pregnancy, there are abnormally low levels of RANKL/RANK in villi and decidua from miscarriage patients. These results suggest that RANKL is a pivotal regulator of maternal-fetal tolerance by licensing $\mathrm{dM} \varphi$ to ensure a successful pregnancy outcome. This observation provides a scientific basis on which a potential therapeutic strategy can be targeted to prevent pregnancy loss.

Cell Death and Disease (2017) 8, e3105; doi:10.1038/cddis.2017.505; published online 12 October 2017

Pregnancy constitutes a major challenge to the maternal immune system, which must tolerate fetal alloantigen encoded by paternal genes. ${ }^{1,2}$ The disturbance of maternal-fetal immune regulation is associated with several complications of human pregnancy, including spontaneous abortion (SA), intrauterine growth restriction (IUGR) and preeclampsia. ${ }^{3-5}$ Accumulating evidence indicates that decidual macrophages $(\mathrm{dM} \varphi)$, the second largest decidual leukocyte population during the first trimester ( 20\%) following decidual NK cells (dNKs, 50-70\%), are involved in several processes required for a successful pregnancy, including trophoblast invasion, as well as tissue and vascular remodeling., ${ }^{6,7}$ However, the mechanisms responsible for $\mathrm{dM} \varphi$ differentiation and polarization at the maternal-fetal interface remain largely unexplored.

Of note, two distinct states of polarized activation of macrophages have been recognized: the classically activated (M1) macrophage phenotype and the alternatively activated (M2) macrophage phenotype. ${ }^{8-10}$ Bacterial moieties such as LPS and TH1 cytokine interferon- $\gamma$ (IFN- $\gamma$ ) polarize macrophages toward the M1 phenotype. These M1 macrophages are characterized by high interleukin (IL)-12 and IL-23 and low IL-10 production, and accordingly can kill intracellular microorganisms and induce Th1 immunity. In contrast, M2 polarization was originally discovered as a response to the Th2 cytokines IL-4 and IL-13, the anti-inflammatory cytokine IL-10, M-CSF, glucocorticoids and immune complexes. They generally share characteristics such as high IL-10 and low IL-12 and IL-23 production, anti-inflammatory and tissue remodeling properties, and scavenging of apoptotic cells and debris, and therefore have been considered to be important regulators of the immune response.

The $\mathrm{dM} \varphi$ were classified as resembling an M2 phenotype. ${ }^{11}$ However, there is still ambiguity with regard to the distinct functions of the $\mathrm{dM} \varphi$ subset.,6,12 Recent research has revealed that first-trimester $\mathrm{dM} \varphi$ can be divided into two distinct subsets, CD209 ${ }^{+}$and CD209- ${ }^{-} \mathrm{MM} \varphi{ }^{6,12}$ In comparison

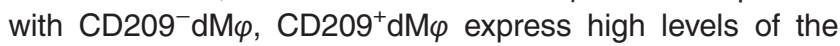
scavenger receptor CD163, the phagocytic receptors CD206 and CD304, and the CD209 ligand ICAM-3, and low levels of CD11c, which are associated with spiral arteriole remodeling. ${ }^{6}$

Receptor activator of NF- $k$ B ligand (TNFSF11, also known as RANKL) and its tumor necrosis factor (TNF)-family receptor RANK are essential regulators of osteoclast differentiation and thereby fundamental aspects of bone physiology, bone remodeling, ${ }^{13,14}$ lymph node formation, ${ }^{15}$ establishment of thymic microenvironment, ${ }^{16}$ mammary gland development during pregnancy ${ }^{17,18}$ and bone metastasis of cancer. ${ }^{19}$ Osteoprotegerin (OPG) is a decoy receptor for RANKL. By binding RANKL, OPG blocks the RANKL-RANK interaction. Osteoclasts are derived from monocyte/macrophage precursors. ${ }^{13}$ However, the role of RANKL in inducing

\footnotetext{
'Laboratory for Reproductive Immunology, Hospital of Obstetrics and Gynecology, Fudan University Shanghai Medical College, Shanghai, People's Republic of China; ${ }^{2}$ Key Laboratory of Reproduction Regulation of NPFPC, SIPPR, IRD, Fudan University, Shanghai, People's Republic of China; ${ }^{3}$ Shanghai Key Laboratory of Female Reproductive Endocrine Related Diseases, Hospital of Obstetrics and Gynecology, Fudan University Shanghai Medical College, Shanghai, People's Republic of China and ${ }^{4}$ Clinical and Translational Research Center, Shanghai First Maternity and Infant Hospital, Tongji University School of Medicine, Shanghai, People's Republic of China

*Corresponding author: M-Q Li or D-J Li, Laboratory for Reproductive Immunology, Hospital of Obstetrics and Gynecology, Fudan University Shanghai Medical College, Shanghai 200011, People's Republic of China. Tel/Fax: +86 21 63457331; E-mail: mqli@fudan.edu.cn or djli@shmu.edu.cn

${ }^{5}$ These authors contributed equally to this work.

Received 27.7.17; revised 01.9.17; accepted 01.9.17; Edited by H-U Simon
} 


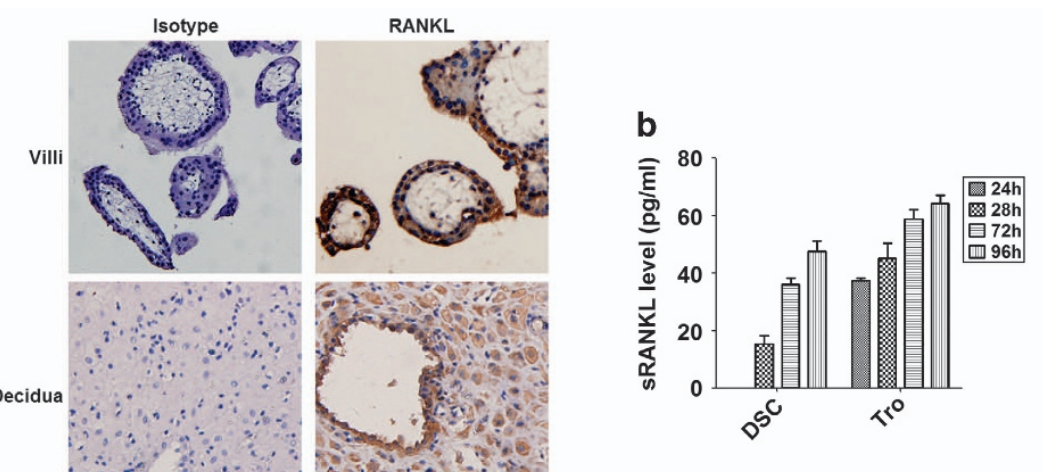

C

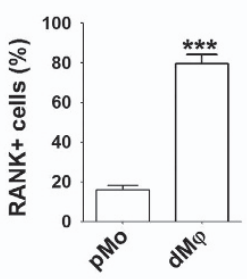

d

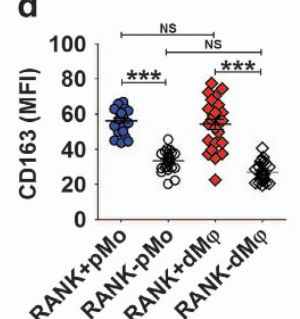

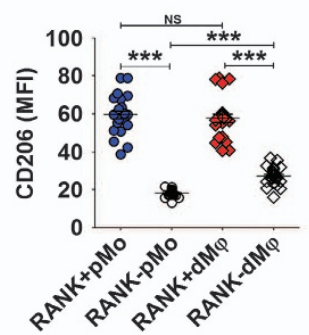

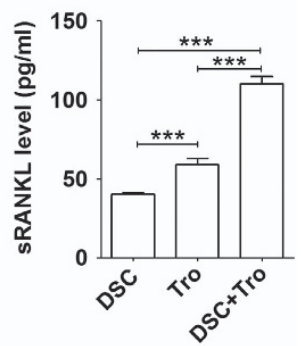

e Isotype
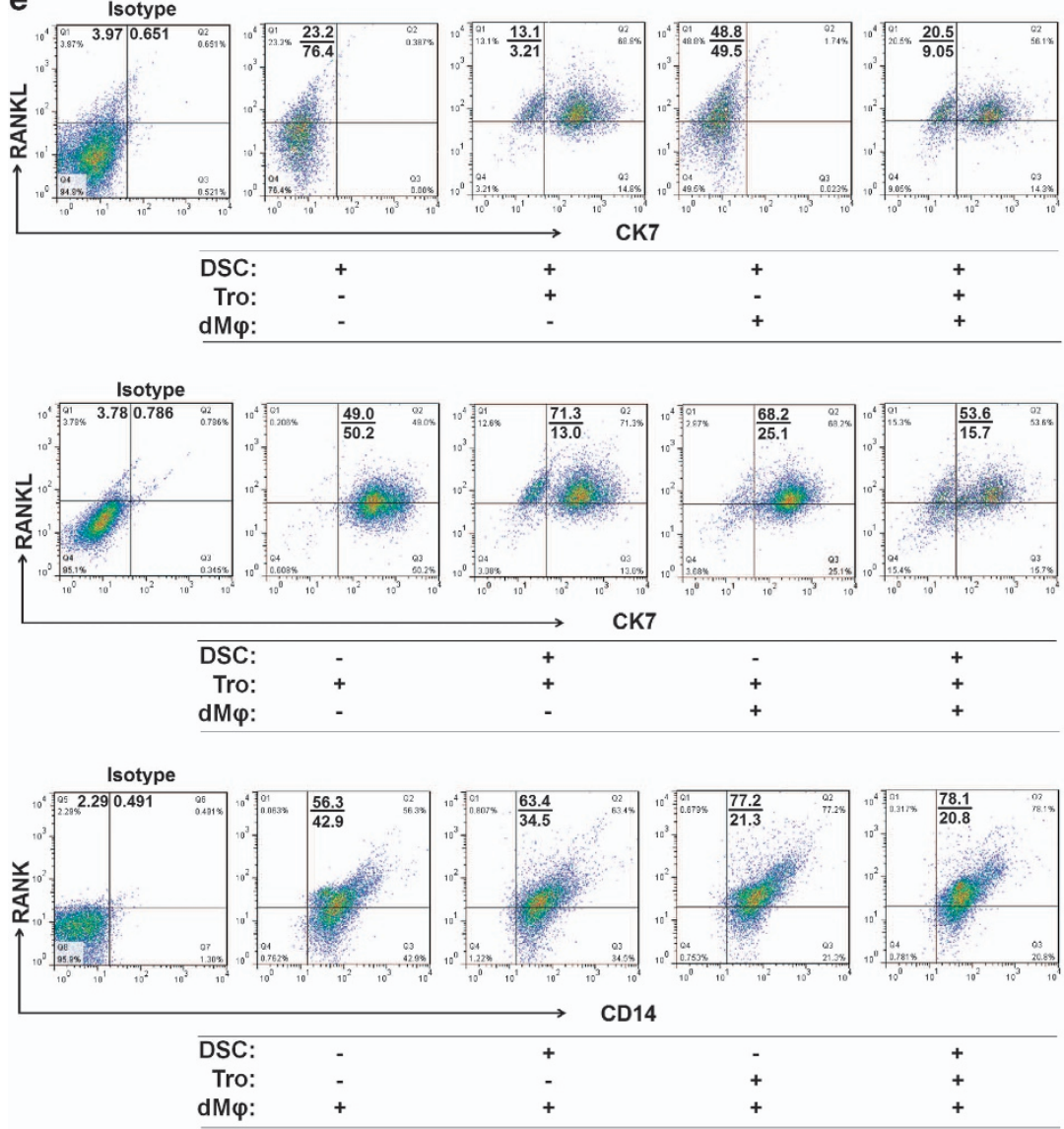
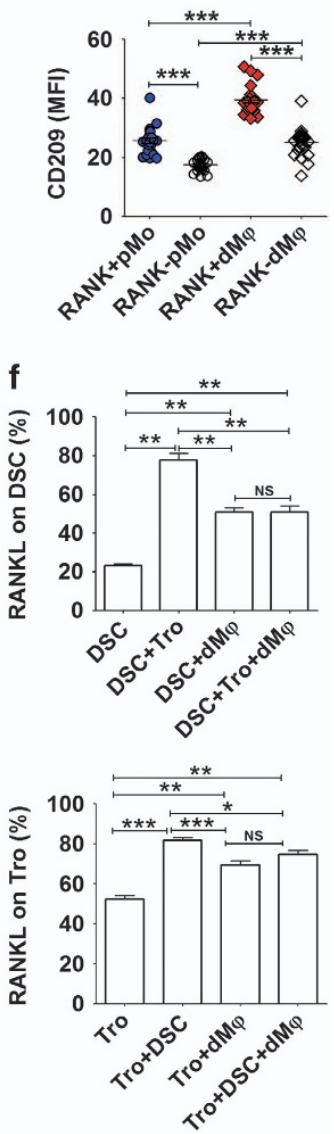

\section{$f$}
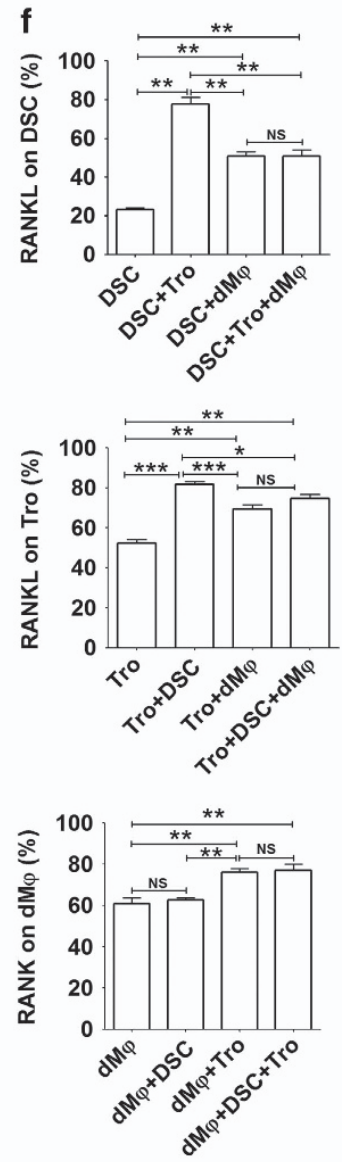
Figure 1 The crosstalk between fetus and mothers leads to high levels of RANKL/RANK expression at the maternal-fetal interface. (a) RANKL expression in villi and decidua of normal pregnancy $(n=12)$ by immunohistochemistry. Original magnification: $\times 200$. (b) RANKL secretion by primary trophoblasts $\left(1 \times 10^{5}\right.$ cells per well) and DSCs $\left(1 \times 10^{5}\right.$ cells per well) $(n=6)$ from normal pregnant women by ELISA after culture for 24-96 $\mathrm{h}$ (left). RANKL production by trophoblasts alone, DSCs alone and the co-culture of trophoblasts and DSCs $(n=6)$ for $48 \mathrm{~h}$ (right). (One-way ANOVA). (c) We isolated primary PBMCs from peripheral blood $(n=6)$ and DLC from deciduas of normal pregnant women $(n=6)$, and then analyzed RANK expression on pMo and dM $\varphi$ from normal pregnant women by labeling with anti-CD14, RANK and CD45 antibodies. (Student's $t$-test). (d) Further analysis of the phenotype of RANK ${ }^{+}$and RANK ${ }^{-}$pMo and dM $\varphi$ from normal pregnant women $(n=24)$ by FCM. (One-way ANOVA). (e and $f$ ) Trophoblasts, DSCs and/ or $\operatorname{dM} \varphi(n=6)$ were co-cultured at a $1: 1: 1$ ratio for $48 \mathrm{~h}$, and then RANKL expression on CK7 $7^{+}$trophoblasts and CK7 ${ }^{-}$DSCs, and RANK on CD14 ${ }^{+}$dM $\varphi$ were evaluated by FCM, respectively. (One-way ANOVA). Tro: human trophoblasts. pMo: human peripheral blood monocytes; dM $\varphi$ : human dM $\varphi$. Data are expressed as the mean \pm S.E.M. ${ }^{*} P<0.05,{ }^{* *} P<0.01$ and ${ }^{* * *} P<0.001$. NS: no statistical difference

macrophage differentiation and functional regulation at the maternal-fetal interface is largely unknown.

In this study, we investigated the effect of RANKL from human embryonic trophoblasts and maternal DSCs on $\mathrm{dM} \varphi$ differentiation and maternal-fetal immune tolerance, and we analyzed the relationship of RANKL production at the interface with miscarriage.

\section{Results}

The crosstalk between fetus and mothers leads to high levels of RANKL/RANK expression at the maternal-fetal interface. To investigate the role of RANKL/RANK signaling at the maternal-fetal interface, we first analyzed the expression of RANKL and found that both embryonic trophoblasts from villi and maternal DSCs from decidua are positive for RANKL in human first-trimester pregnancy (Figures 1a and b). As observed by immunohistochemistry, RANKL expression was located both in cell membrane and cytoplasm (Figure 1a). Similar results for RANKL expression levels were obtained by ELISA and flow cytometry (FCM) of the isolated trophoblasts and DSCs. In comparison with DSCs, trophoblasts secreted more soluble RANKL (sRANKL) and expressed higher level of membrane RANKL (mRANKL), and the co-culture of trophoblasts and DSCs produced higher levels of sRANKL (Figure 1b).

To identify target cells of RANKL at the maternal-fetal interface, we analyzed the expression of RANK in decidual leukocyte cells (DLCs) primarily isolated from human decidual tissue. Among DLCs, the percentage of RANK ${ }^{+}$cells in CD45 ${ }^{+}$ $\mathrm{CD} 14^{+} \mathrm{dM} \varphi$, as well as decidual NKT and $\mathrm{CD}^{+} \mathrm{T}$ cells was $\sim 80 \%$ (Supplementary Figure 1a), which was 4.45-fold higher than that of $\mathrm{CD} 14^{+}$monocyte cells from peripheral blood (pMo) (Figure 1c).

Interestingly, further phenotypic analysis showed that both $\mathrm{RANK}^{+} \mathrm{pMo}$ and $\mathrm{RANK}^{+} \mathrm{dM} \varphi$ expressed higher levels of M2polarized macrophages markers (10) (the scavenger receptor CD163, the phagocytic receptors CD206 and CD209) and M1 co-stimulatory molecules CD80, CD86, HLA-DR, CD11c and cytokine IL-12p40, compared with RANK ${ }^{-}$cells (Figure $1 \mathrm{~d}$ and Supplementary Figure 1b). These significant differences in M2 phenotype between RANK ${ }^{+}$pMo and RANK ${ }^{-}$pMo was maintained and even augmented in $\mathrm{dM} \varphi$. Conversely, the $\mathrm{M} 1$ advantages possessed by $\mathrm{RANK}^{+} \mathrm{pMo}$ gradually weakened in $\mathrm{RANK}^{+} \mathrm{dM} \varphi$. In comparison with $\mathrm{RANK}^{-} \mathrm{dM} \varphi$, the expression pattern of $\mathrm{RANK}^{+}$in $\mathrm{dM} \varphi$ suggests that RANK signaling may regulate the differentiation and function of $\mathrm{dM} \varphi$.

Embryonic extravillous trophoblasts (EVT) are in direct contact with maternal DSCs and DLCs, and the interaction between these cell subsets has an important role in maintaining maternal-fetal tolerance, which is required for a successful pregnancy. ${ }^{3}$ To investigate this intercellular crosstalk, we cocultured primary human trophoblasts, DSCs and $\mathrm{dM} \varphi(\mathrm{T}+\mathrm{D}$ $+\mathrm{M})$. FCM analysis revealed markedly increased expression of RANKL in trophoblasts and DSCs and RANK on $\mathrm{dM} \varphi$ in the $\mathrm{T}+\mathrm{D}+\mathrm{M}$ co-culture (Figures $1 \mathrm{e}$ and $\mathrm{f}$ ). The percentage of $\mathrm{RANK}^{+} \mathrm{dM} \varphi$ was reduced to $60 \%$ after culture alone for $48 \mathrm{~h}$. The presence of embryonic trophoblasts in the absence of DSCs led to a frequency of $80 \% \mathrm{RANK}^{+} \mathrm{dM} \varphi$ (Figures 1e and f). Taken together, these data suggest that embryonic trophoblasts may have a crucial role in the regulation of maternal $\mathrm{dM} \varphi$ differentiation and function through the RANKL-RANK interaction.

RANKL from trophoblasts and DSCs triggers M2 differentiation of $\mathrm{dM} \varphi$ and Th2 bias. To investigate the potential effect of RANKL on $\mathrm{dM} \varphi$, we directly co-cultured the purified $\mathrm{CD} 14^{+} \mathrm{dM} \varphi$ with RANKL-overexpressed DSCs and JEG-3 cells (human placental choriocarcinoma cell line) (RANKL ${ }^{+} D$ $+J)$. Compared with control DSCs and JEG-3 cells (Ctrl-D+J), RANKL ${ }^{+} \mathrm{D}+\mathrm{J}$ resulted in the elevation of CD206, CD209, CD163 and IL-10 and the decline of IFN- $\gamma$, IL-12/23p40, $\mathrm{CD} 80$ and $\mathrm{CD} 86$ in $\operatorname{CD}_{14}^{+} \mathrm{dM} \varphi$ (Figures $2 a$ and $b$ ). In contrast, blocking the RANKL-RANK interaction with a neutralizing antibody against RANKL ( $a$-RANKL) or OPG protein could reverse the expression of CD80 and CD86 and the release of IL-10, IL-12p40 and IL-23 induced by RANKL of trophoblasts and DSCs (Supplementary Figure 2).

RANKL participates in the regulation of monocyte migration by inducing the secretion of chemokine, such as CCL22 and CCL2. ${ }^{20,21}$ However, current results show that RANKL was not involved in the regulation of the M2-recruited chemokines CCL17, CCL22 and CCL24 and the M1-recruited chemokines CXCL9 and CXCL10 of $\mathrm{dM} \varphi$ (data not shown). We have previously reported that RANKL stimulates DSCs to secrete the chemokine CCL2. ${ }^{22}$ Our study suggests that RANKL may recruit peripheral monocyte to decidua, and further polarize them toward $\mathrm{M} 2 \mathrm{dM} \varphi$.

Evidence suggests that the RANKL-RANK interaction increases macrophage/dendritic cell (DC) survival and enhances the induction of T-cell response. ${ }^{23-25}$ However, published evidence shows that RANKL-mediated modulation of DCs in mucosal tissues increases the number of $\mathrm{CD}^{+}$ $\mathrm{CD}^{+} 5^{+}$regulatory $\mathrm{T}$ (Treg) cells and promotes immunosuppressive activity toward foreign antigens, such as food or commensal bacteria in the intestines. ${ }^{26-28}$ However, the molecular mechanism underlying RANKL on M $\varphi s$ and DCs remains unclear. We further investigated the regulation of these RANKL-instructed $\mathrm{dM} \varphi$ on the differentiation of decidual 
a

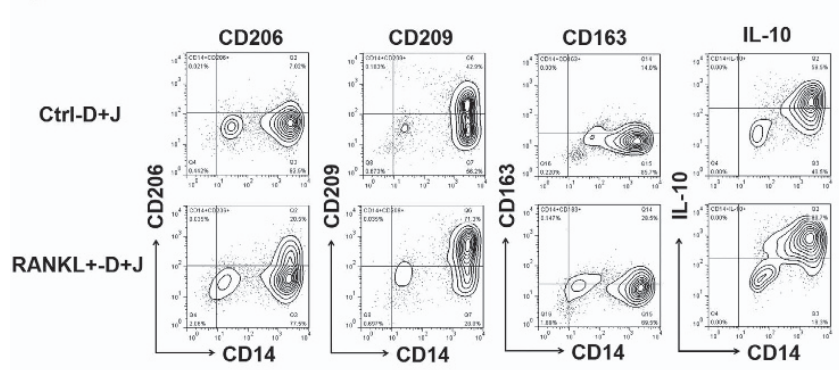

C

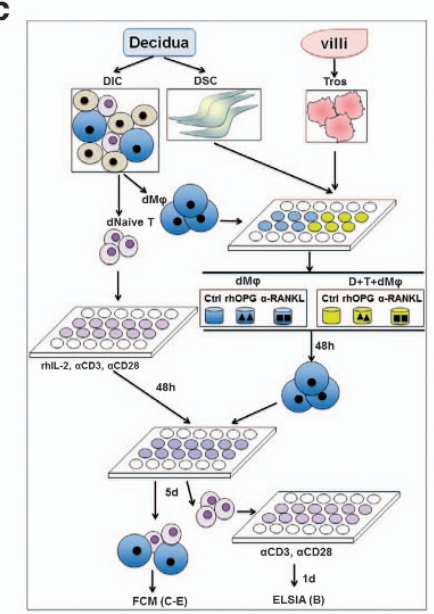

d

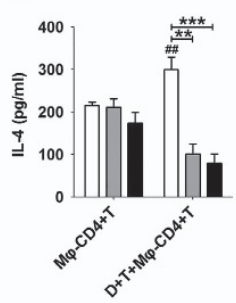

b
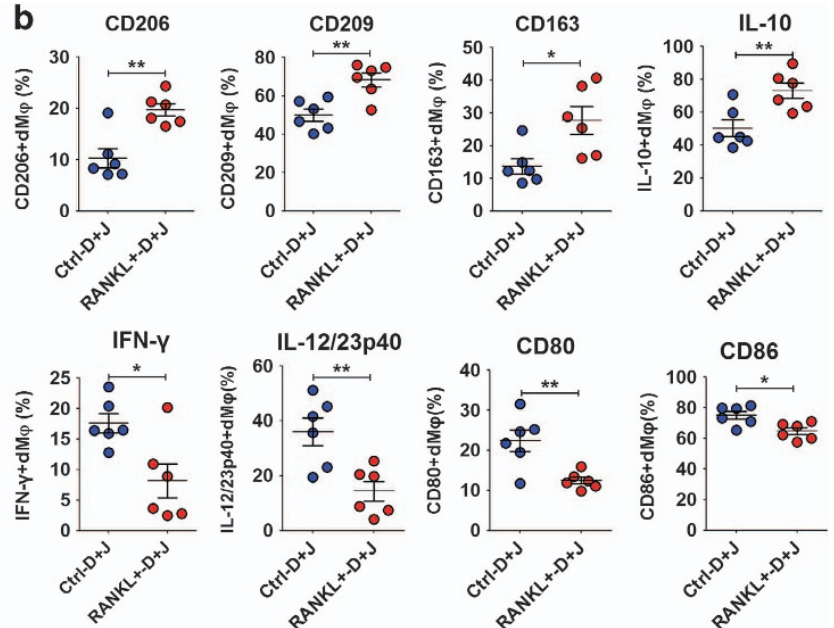

IL-12/23p40
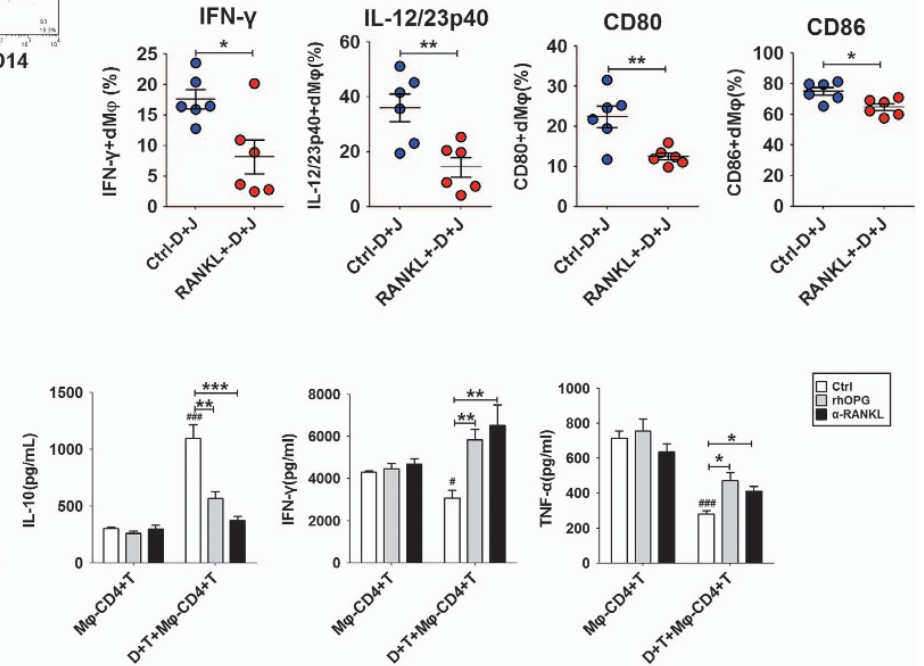
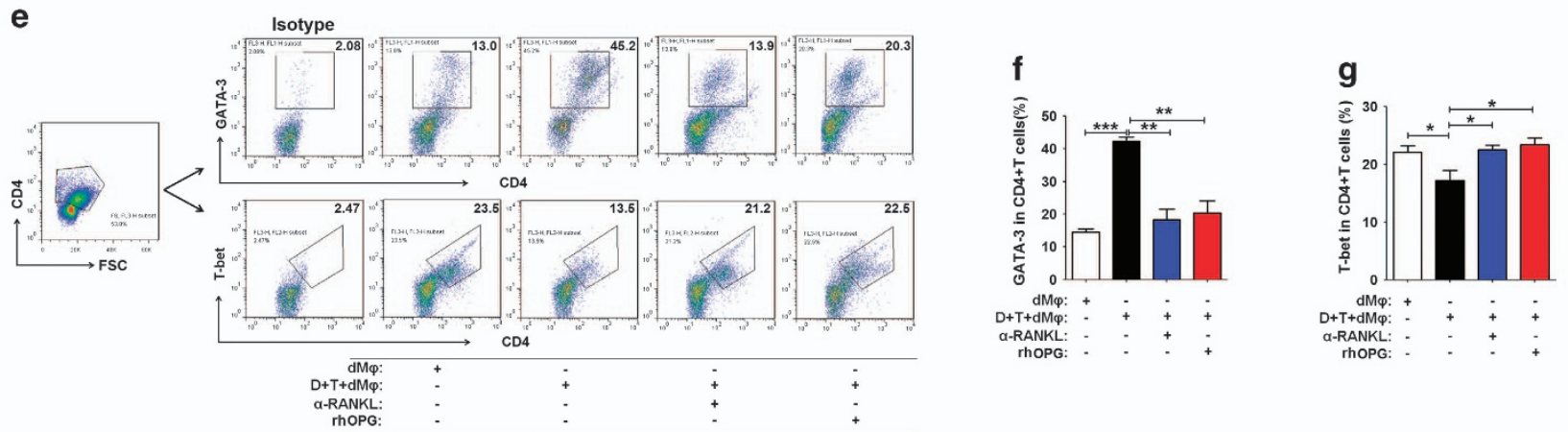

Figure 2 RANKL from trophoblasts and DSCs triggers M2 differentiation of $\mathrm{dM} \varphi$ and Th2 bias. (a and $\mathbf{b})$ We co-cultured dM $\varphi(n=6)$ with RANKL-overexpressed or control DSCs and JEG-3 cells at a 1:1 : 1 ratio for 48h, and then the expression levels of CD206, CD209, CD163, IL-10, CD80, CD86, IFN- $\gamma$ and IL-12/23p40 were assessed in dM $\varphi$. (Student's t-test). (c-g) After $48 \mathrm{~h}$ of culture with or without trophoblasts and DSCs and treatment with or without recombinant human OPG protein (rhOPG, $100 \mathrm{ng} / \mathrm{ml}$ ) or antihuman RANKL neutralizing antibody ( $\alpha$-RANKL, $5 \mu \mathrm{g} / \mathrm{ml}), \operatorname{CD} 14^{+} \mathrm{dM} \varphi(n=6)$ were collected and co-cultured with autologous decidual naive T cells at ratios of $1: 1$ (c). The decidual naive T cells had been previously activated with anti-CD3 $(5 \mu \mathrm{g} / \mathrm{ml})$, anti-CD28 $(1 \mu \mathrm{g} / \mathrm{ml})$ and rhlL-2 $(20 \mathrm{U} / \mathrm{ml})$ for 3 days, and then collected. After 5 days of co-culture, the expression of GATA-3 and T-bet in CD4 ${ }^{+} T$ cells $(\mathrm{e}-\mathrm{g})$ were analyzed by FCM; alternately, these $\mathrm{CD} 4^{+} \mathrm{T}$ cells were collected and reactivated with anti-CD3 and anti-CD28 alone for another $24 \mathrm{~h}$, and then the secretion levels of IL-4, IL-10, TNF- $\alpha$ and IFN- $\gamma$ (d) were analyzed by ELISA. (One-way ANOVA). dM $\varphi$ : human dM $\varphi$; Ctrl-D+J: control DSCs and JEG-3 cells; RANKL ${ }^{+} D+J$ : RANKL-overexpressed DSCs and JEG-3 cells; M $\varphi$-CD4+T: co-culture of ctrl dM $\varphi$ and naive T cells; D+T+CD4+T: co-culture of dM $\varphi$ pre-cultured with DSCs and trophoblasts and naive T cells. Data are expressed as the mean \pm S.E.M. ${ }^{\star} P<0.05$, ${ }^{\star *} P<0.01$ and ${ }^{\star \star *} P<0.001 .{ }^{\#} P<0.05$, ${ }^{\# \#} P<0.01$ and ${ }^{\# \# \#} P<0.001$ versus ctrl $\mathrm{M} \varphi$ - $\mathrm{CD} 4+\mathrm{T}$

naive T cells. After pre-culture with trophoblasts and DSCs, we collected $\mathrm{dM} \varphi$, and then co-cultured them with decidual naive $T$ cells for 5 days (Figure 2c). We observed that either $a$-RANKL or OPG abolished the stimulatory effect on the IL-10 and Th2 transcription factor GATA-3 and the inhibitory impact on tumor necrosis factor- $a$ (TNF- $\alpha$ ) and Th1 transcription factor T-bet in $\mathrm{CD} 4^{+} \mathrm{T}$ cells mediated by $\mathrm{dM} \varphi$ pre-treated with trophoblasts and DSCs $(\mathrm{D}+\mathrm{T}-\mathrm{dM} \varphi)$ (Figures $2 \mathrm{~d}-\mathrm{g})$. In addition, blocking RANKL in the T+D+M co-culture further inhibited IL-4 secretion but stimulated IFN- $\gamma$ production of $\mathrm{CD}^{+} \mathrm{T}$ cells (Figures 2d-g). However, RANKL-instructed $\mathrm{dM} \varphi$ had no effect on decidual Treg cell differentiation (data not shown).

As a potent inducer of decidual $\mathrm{M} 2 \mathrm{M} \varphi,{ }^{6}$ the increased IL-10 in $\operatorname{dM} \varphi$ and decidual $\mathrm{CD} 4^{+} \mathrm{T}$ cells induced by RANKL may further amplify the impact of RANKL on M2 polarization of $\mathrm{dM} \varphi$. These data provide strong evidence that RANKL is 
expressed at the maternal-fetal interface, and support the presence of a positive regulatory loop between trophoblasts and $\mathrm{dM} \varphi$ to induce maternal-fetal immune tolerance during pregnancy.

The effect of RANKL on $\mathrm{dM} \varphi$ is dependent on the activation of the Akt/STAT6-Jmjd3/IRF4 signaling pathway. Of note, mRANKL or sRANKL cleaved by matrix metalloproteinases (MMPs) or a disintegrin and metalloproteases (ADAMs) binds RANK and then mainly activates the nuclear factor kappa-light-chain-enhancer of activated $B$ cells (NF-KB) pathway to control osteoclastogenesis through adaptor molecules such as TRAFs and Gab2, ${ }^{29-31}$ thus regulating osteoimmunology by pro- and anti-inflammatory effects on the immune system. ${ }^{32}$ To identify the downstream pathway through which RANKL drives M2 polarization during pregnancy, we assessed the impact of RANKL on different signaling pathways in $\mathrm{dM} \varphi$. $\mathrm{T}+\mathrm{D}+\mathrm{M}$ led to changes in the phosphorylation of several signaling pathways such as Akt, NF-kBp65 and c-Jun N-terminal kinases (JNK) (data not shown), suggesting that several pathways should be involved in the functional regulation on $\mathrm{dM} \varphi$. Notably, $a-\mathrm{RANKL}$ or OPG specifically inhibited the activation of Akt and STAT6 (a master regulator of $\mathrm{M} 2$ genes in mice downstream of IL-4R) ${ }^{33-35}$ in $\mathrm{dM} \varphi$ (data not shown). Culture with RANKL ${ }^{+}-$ $D+J$ gave rise to the increased level of Akt and STAT6 phosphorylation in $\mathrm{dM} \varphi$ compared with the culture with Ctrl-D $+\mathrm{J}$ (Figures $3 \mathrm{a}$ and b). The Akt signal inhibitor (Akti) LY294002 partly reversed the level of STAT6 phosphorylation (Figure 3c).

Compared with the control, the release of $\mathrm{IL}-10$ and $\mathrm{IL}-12 \mathrm{p} 40$ and IL-23 by $\mathrm{dM} \varphi$ increased and decreased, respectively, under co-culture with $R A N K L^{+}-D+J$, and these effects could be clearly impaired by Akti or STAT6 inhibitor (STAT6i) AS1517499 ${ }^{35}$ treatment (Figure 3d). Interestingly, treatment with STAT6i resulted in M1 differentiation and Th1 bias in the mice uterus in vivo (Figure $3 e$ ). Therefore, these data suggest that RANKL induces M2 differentiation by activating the Akt/STAT6 signaling pathway.

It has been reported that Jmjd3 regulates M2 polarization by inhibiting the transcription of typical $\mathrm{M} 1$-associated genes and inducing IRF4. ${ }^{36-38}$ Unlike IRF5, ${ }^{39}$ IRF4 has been recognized as an essential transcription factor for M2 polarization and the expression of M2 signature genes such as Arg1, Ym1 and Fizz1 in mice. ${ }^{40}$ We investigated the effects of RANKL on these transcription factors. Blocking RANKL resulted in a marked decrease in Jmjd3 and IRF4 but not IRF5 transcription in the co-culture of $\mathrm{D}+\mathrm{T}-\mathrm{dM} \varphi$ (Supplementary Figure 3). Conversely, there was an increase in the transcription of $J$ mjd3 and IRF4 in $\mathrm{RANKL}^{+}-\mathrm{D}+\mathrm{J}-\mathrm{dM} \varphi$, and both Akti and STAT6i treatment could partially abrogate these effects on Jmjd3 and IRF4 induced by RANKL in vitro (Figure 3f). Similarly, both Jmjd3 selective inhibitor (JMJD3i) GSK J4 HCl and STAT6i treatment could downregulate IRF4 expression in $\mathrm{uM} \varphi$ (Figure $3 \mathrm{~g}$ ). Furthermore, treatment with JMJD3i also led to $\mathrm{M} 1$ differentiation (Figure $3 \mathrm{~h}$ ) and a Th1 bias (Figure 3i) in mouse uterus. Taken together, these results place RANKL upstream in the Akt/STAT6-Jmjd3/IRF4 signaling cascade involved in $\mathrm{M} 2$ polarization of $\mathrm{dM} \varphi$.
Downregulation of RANKL expression leads to murine decidual $\mathbf{M} \boldsymbol{\varphi}$ dysfunction and fetal loss. In comparison with non-pregnant mice, uterine $\mathrm{M} \varphi(\mathrm{uM} \varphi)$ from pregnant mice had a high level of RANK (Supplementary Figure 4). To further explore the role of absent RANKL at the maternalfetal interface during the differentiation of $\mathrm{M} \varphi$ in vivo, we first evaluated RANKL/RANK expression in the uterus in normal pregnancy and abortion-prone matings. In $\mathrm{CBA} / \mathrm{J} \times \mathrm{DBA} / 2$ matings as an abortion-prone model, decreased RANK expression was detected in $\mathrm{F} 4 / 80^{+} \mathrm{uM} \varphi$ on gestational days 5 and 9 , compared with $\mathrm{CBA} / \mathrm{J} \times \mathrm{BALB} / \mathrm{c}$ matings as a mouse model of normal pregnancy (Figure $4 a$ ).

Interestingly, $\mathrm{uM} \varphi$ in mice are divided into two subsets: $\mathrm{F} 4 / 80^{+} \mathrm{MHClI}^{\mathrm{hi}}$ and $\mathrm{F} 4 / 80^{+} \mathrm{MHClI}^{\mathrm{lo}}$. These two subsets differentially express $C D 163$ and Mrc1, which may represent

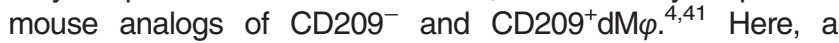
characteristic M1 rather than M2 phenotype was also observed in $\mathrm{uM} \varphi$ from $\mathrm{CBA} / \mathrm{J} \times \mathrm{DBA} / 2$ abortion-prone matings (Figure $4 \mathrm{a}$ ). Similarly, uM $\varphi$ in RANKL knockout ( $\mathrm{RANKL}^{-/-}$) pregnant mice had low levels of CD206, CD209 and IL-10, and high levels of CD80 and CD86 compared with wild-type (WT) pregnant mice (Figure 4b). In comparison with WT group, there was a Th1 bias in the uterus of RANKL ${ }^{-1}$ pregnant mice (Figure 4c). Furthermore, there were low levels of Akt and STAT6 phosphorylation, Jmjd3 and IRF4 in $\mathrm{uM} \varphi$ of RANKL ${ }^{-1}$ pregnant mice compared with WT group (Figures 4d-f).

To investigate the influence of RANKL/RANK signaling on outcome of pregnancy in vivo, we evaluated embryoabsorbing level between $\mathrm{RANKL}^{-1-}$ and WT pregnant mice. The embryo-absorbing site could be macroscopically distinguished as hemorrhagic spots and necrosis at late gestation (gestational day 14, Figure $4 \mathrm{~g}$ ). The $\mathrm{RANKL}^{-/}$-mice were more susceptible to fetal loss than WT mice (Figures $4 \mathrm{~g}$ and h). These findings provide evidences of a key role of RANKL in the regulation of $\mathrm{dM} \varphi$ differentiation and function, promoting maternal-fetal tolerance to support normal pregnancy. Abnormal suppression of RANKL expression contributes to $\mathrm{uM} \varphi$ dysfunction and fetal loss in vivo.
Adoptive transfer of $\mathrm{RANK}^{+} \mathrm{M} \varphi$ relieves murine embryo absorption induced by $\mathrm{M} \varphi$ depletion. The depletion of $\mathrm{M} \varphi$ in pregnant mice using Clodronate Liposomes (Supplementary Figures $5 \mathrm{a}$ and $\mathrm{b}$ ) led to a significant decrease in RANKL in uterine DSCs (uDSCs) and placental trophoblasts (pTros) (Supplementary Figure 5c), suggesting that $\mathrm{dM} \varphi s$ are involved in the maintenance of high levels of RANKL at the maternal-fetal interface. To provide insight into the role of RANKL-instructed $\mathrm{dM} \varphi$ in maternal-fetal immune regulation and pregnancy outcome in vivo, we evaluated the effect of $\mathrm{dM} \varphi$ depletion and adoptive transfer of $\mathrm{RANK}^{+} \mathrm{M} \varphi$ on these processes. Next, we isolated $\mathrm{RANK}^{+}$and $\mathrm{RANK}^{-} \mathrm{M} \varphi \mathrm{s}$ from mouse spleen and observed that these $\operatorname{RANK}^{+} \mathrm{M} \varphi \mathrm{s}$, like human RANK ${ }^{+}$pMo, had high levels of M1 and M2 phenotype molecules (Supplementary Figure 6a). To investigate the process of $\mathrm{RANK}^{+} \mathrm{M} \varphi$ differentiation in the uterus in vivo, we labeled these $\mathrm{RANK}^{+}$and $\mathrm{RANK}^{-} \mathrm{M} \varphi \mathrm{s}$ with $\mathrm{PKH}-67$ and transferred them to $\mathrm{M} \varphi$-deleted pregnant mice (Figure $5 \mathrm{a}$ and Supplementary Figure 6b). RANK ${ }^{+} M \varphi s$ with high CCR2 were preferentially recruited to the uterus (Supplementary Figure 6c). In comparison with the $\mathrm{PKH}-67-\mathrm{RANK}^{-} \mathrm{M} \varphi$ 
a

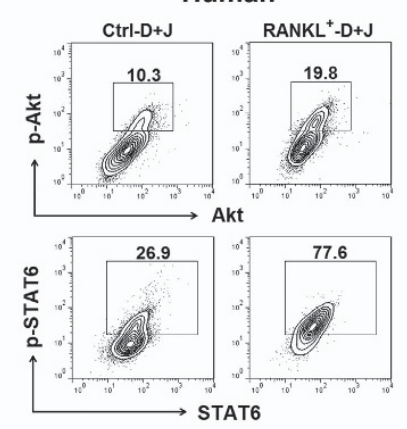

d

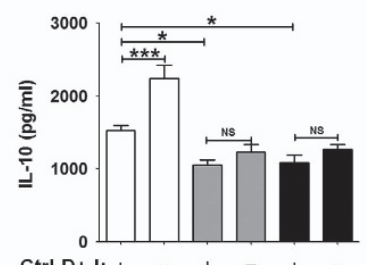

Ctr b

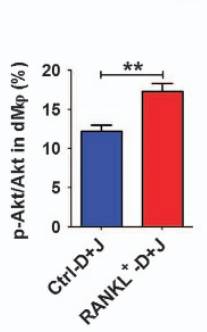

Human

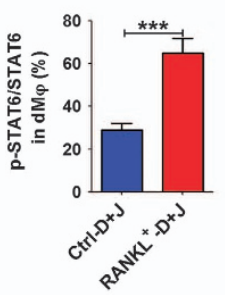

Human

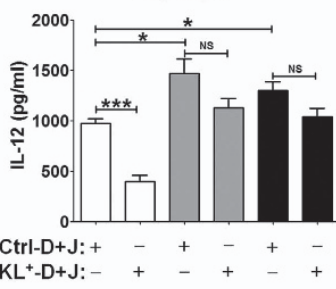

C

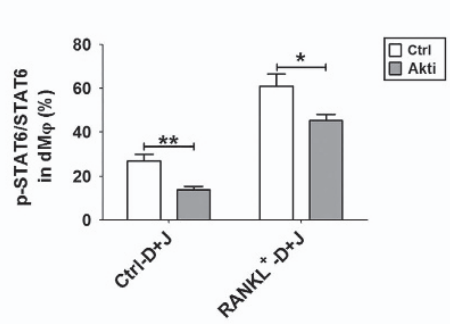

e

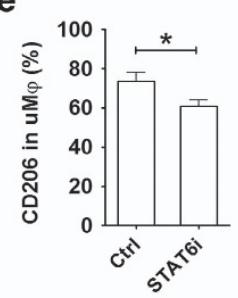

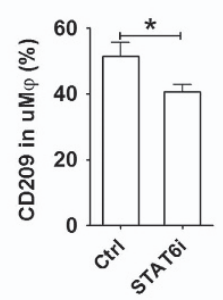
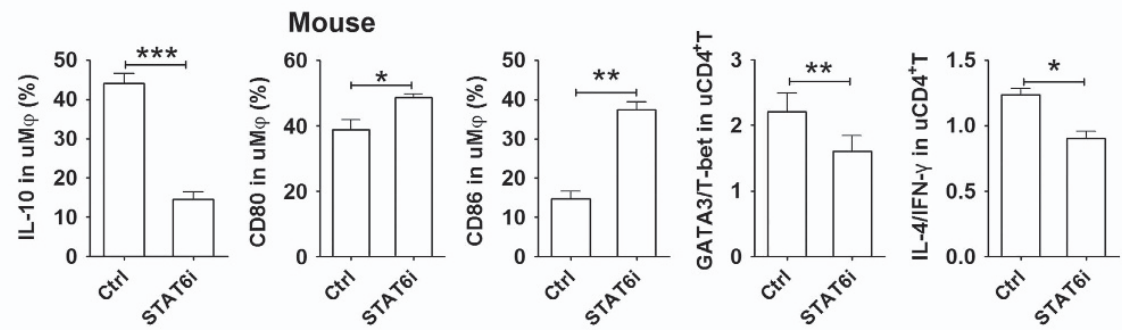

f

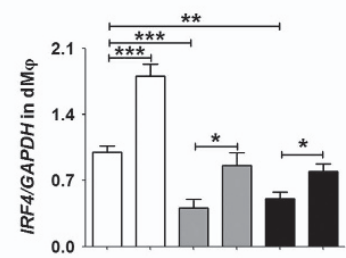

Ctrl-D+J: +

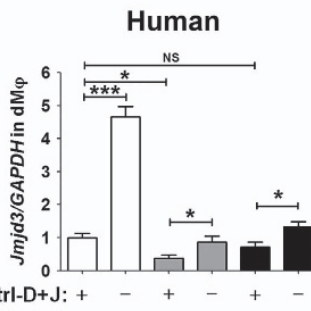

RANKL ${ }^{+}-\mathrm{D}+\mathrm{J}: \mathrm{C}_{-}+\mathrm{C}_{-}+$

Ctrl-D+J: RANKL+-D+J: -
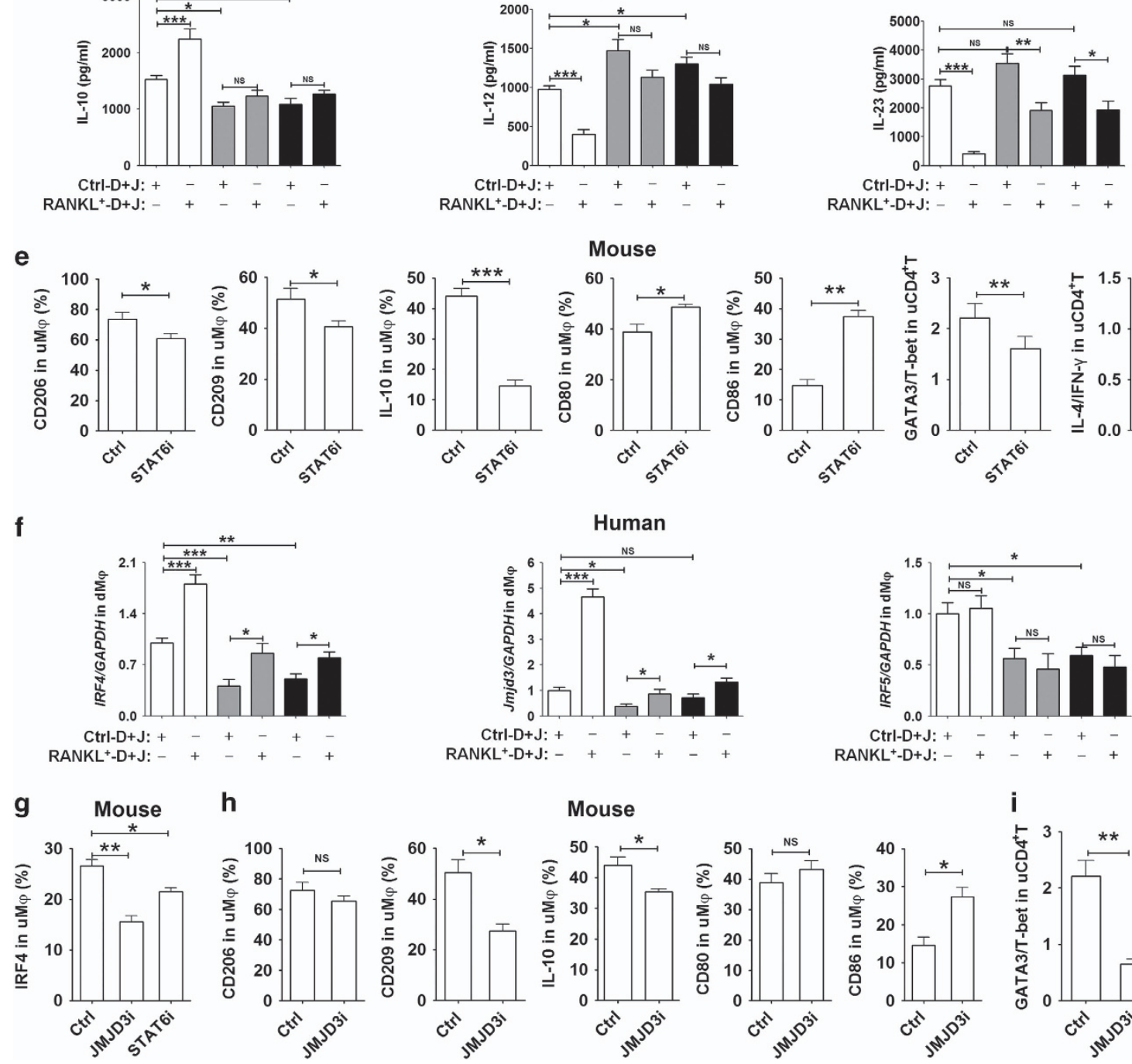

Ctri

STAT6i

Ctrl-D+J: + RANKL ${ }^{+}-\mathrm{D}+\mathrm{J}: \mathrm{C}_{-}++_{-}+$

Figure 3 RANKL induces dM $\varphi$ differentiation by activating the Akt/STAT6-Jmjd3/RF4 signaling pathway. (a-c) dM $\varphi$ were co-cultured with RANKL $D+J$ or Ctrl-D+J at a $1: 1: 1$ ratio, and treated with or without $10 \mu \mathrm{M}$ Akt signaling inhibitor (Akti) Ly294002 for $24 \mathrm{~h}$. The intracellular phosphorylation level of Akt and or STAT6 in dM $\varphi(n=5)$ was then analyzed by FCM. (d) The secretion level of IL-10, IL-12p40 and IL-23 in the co-culture of dM $\rho$ and RANKL'-D+J or Ctrl-D+J (at a $1: 1: 1$ ratio), which was treated with or without Akti (10 $\mu \mathrm{M}$ ) or the STAT6 signaling inhibitor (STAT6i) AS1517499 (21 nM) for $48 \mathrm{~h}$. (One-way ANOVA). (e) After intraperitoneal injection of STAT6i (200 $\mu$ lat the concentration of $2 \mathrm{mg} / \mathrm{kg}$ ) in pregnant C57BL/6 mice ( $n=5$ mice per group) at day 4 , the levels of CD206, CD209, IL-10, CD80 and CD86 in uM $\varphi$, and the ratios of GATA-3 to T-bet and IL-4 to IFN- $\gamma$ in uCD4 ${ }^{+} T$ cells at day 10 were detected by FCM. (Student's t-test). (f) The transcription levels of IRF4, Jmid3 and IRF5 in dM $\varphi$ treated as described in Figure 3d. (One-way ANOVA). (g) After intraperitoneal injection of STAT6i or the Jmjd3 selective inhibitor (JMJD3i, $200 \mu$ at a concentration of $4.48 \mathrm{mg} / \mathrm{kg}$ ) GSK J4 HCl in pregnant C57BL/6 mice ( $n=5$ mice per group) at day 4 , the IRF4 level in uM $\varphi$ cells at day 10 was detected. ( $\mathrm{h}$ and i) After intraperitoneal injection of JMJD3i in pregnant C57BL/6 mice ( $n=5$ mice per group) at day 4, the levels of CD206, CD209, IL-10, CD80 and CD86 in uM $\varphi$, and the ratios of GATA-3 to T-bet and IL-4 to IFN- $\gamma$ in $\mathrm{UCD} 4^{+} \mathrm{T}$ cells at day 10 were detected by FCM. (Student's $t$-test). $\mathrm{dM} \varphi$ : human dM $\varphi$; uM $\varphi$ : mouse uterus macrophages. Data are expressed as the mean \pm S.E.M. ${ }^{*} P<0.05,{ }^{* *} P<0.01$ and ${ }^{* *} P<0.001$ 
transferred group, $\mathrm{PKH}-67-\mathrm{RANK}^{+} \mathrm{M} \varphi$ recruited to uterus presented high levels of CD206 and CD209, and low levels of CD86 and similar levels of CD80 (Figure 5b). In addition, the transfer of $\mathrm{PKH}-67-\mathrm{RANK}^{+} \mathrm{M} \varphi$ led to a Th2 bias in mouse uterus (Figure $5 \mathrm{c}$ ), an increase in Akt and STAT6 activation (Figure $5 \mathrm{~d}$ ), and a high level of IRF4 (Figure $5 \mathrm{e}$ ) in $\mathrm{uM} \varphi$.

Subsequently, we observed that $\mathrm{M} \varphi$ depletion caused a significant increase in fetal loss (Figures $5 f$ and $g$ ). To further identify the role of RANKL-instructed $M \varphi$ in ameliorating fetal loss, we transferred $\mathrm{RANK}^{+}$or $\mathrm{RANK}^{-} \mathrm{M} \varphi$ to $\mathrm{M} \varphi$-deleted pregnant mice and found that adoptive transfer of $\mathrm{RANK}^{+} \mathrm{M} \varphi$ could significantly relieve the murine embryo absorption induced by $\mathrm{M} \varphi$ depletion (Figures $5 \mathrm{~h}$ and i).

The suppression of RANKL/RANK expression and $\mathrm{dM} \varphi$ dysfunction in human miscarriage. The imbalance of maternal-fetal immunoregulation has been previously reported in pregnancy complications ${ }^{3-5}$ such as miscarriage, preeclampsia and IUGR. Therefore, we evaluated RANKL/ RANK expression in the tissue from patients with miscarriage during the first trimester. In comparison with normal pregnancy, we observed a decrease in RANKL expression in trophoblasts and DSCs (Figure 6a), as well as reduced RANK expression on $\mathrm{CD} 14^{+} \mathrm{dM} \varphi$ (Figures $6 \mathrm{~b}$ and $\mathrm{c}$ ) in miscarriage, accompanied by a decreased frequency of $\mathrm{dM} \varphi$ with an M2 phenotype and an increase in the M1 phenotype (Figures $6 \mathrm{~d}$ and $\mathrm{e}$ ). Taken together, the suppression of RANKL/RANK signaling may result in $\mathrm{dM} \varphi$ dysfunction and further trigger miscarriage during the first trimester.

\section{Discussion}

Collectively, we have demonstrated that RANKL derived from embryonic trophoblasts and maternal DSCs drives $\mathrm{dM} \varphi$ polarization toward an M2 phenotype by activating Akt/STAT6 signaling and enhancing the transcription of IRF4 and Jmjd3; finally, it contributes to the formation and maintenance of maternal-fetal tolerance by further inducing Th2 bias (Figure 7). This maternal-fetal dialog mediated by RANKL guarantees a smooth gestation by creating a tolerant microenvironment. The process of maternal-fetal tolerance formation is not only derived from a maternal behavior for immune adaptation to pregnancy but also, most importantly, the fetus can instruct the mother's immune system to adapt to it.

In comparison with the peripheral, the M2 phenotype advantage of $\mathrm{RANK}^{+} \mathrm{dM} \varphi$ becomes more prominent in the decidua. The levels of $M 1$ phenotype markers in $\operatorname{RANK}^{+} \mathrm{dM} \varphi$ are also higher than those in $\mathrm{RANK}^{-} \mathrm{dM} \varphi$, but still very low, similar to the level of RANK ${ }^{-}$pMo. Similarly, it has been reported that both $\mathrm{CD}_{209^{+}}$and $\mathrm{CD} 209^{-} \mathrm{dM} \varphi$ stimulate the release of proinflammatory cytokines such as IL- 6 and TNF- $a$ after LPS stimulation in vitro. ${ }^{12}$ These data emphasize the complexity of $\mathrm{dM} \varphi$ biology. During normal pregnancy, the M2 advantage of $\mathrm{dM} \varphi$ at the maternal-fetal interface is relative and mainly depends on the local microenvironment. This advantage may be disrupted by intrauterine infection and lead to an M1 advantage to limit infection. The expression of proinflammatory molecules in $\mathrm{dM} \varphi$ may align more with the theory that immune activation is required to facilitate trophoblast invasion and implantation, as well as the establishment of fetal-maternal tolerance during the first trimester.

The human maternal-fetal interface is characterized by intimate contact between the maternal decidua and extravillous cytotrophoblast cells that invade the decidua. Trophoblasts can influence the maternal immune system during pregnancy by expressing soluble and cell surface molecules, such as HLA-G, ${ }^{42,43}$ IDO $^{44}$ and anti-inflammatory cytokines. ${ }^{45-47}$ These molecules limit the proliferation and activation of $T$ cells, antigenpresenting cells and NK cells in decidua. In our present study, we found that the crosstalk between embryonic trophoblasts and maternal DSCs and $\mathrm{dM} \varphi$ contributes to the accumulation of RANKL expression at the maternal-fetal interface. RANKL expressed by trophoblasts and DSCs induces M2 differentiation of $\mathrm{dM} \varphi$ and further drives the Th2 bias, suggesting that RANKL/ RANK signaling has a critical role in $\mathrm{dM} \varphi$ differentiation and maternal-fetal tolerance. It is noteworthy that trophoblasts upregulate RANK expression specifically on $\mathrm{dM} \varphi$. These findings further highlight the core role of trophoblasts in $\mathrm{dM} \varphi$ differentiation regulation. Rather than traditional NF- $K B$ signaling under the RANKL/RANK axis, we found that Akt/STAT6-Jmjd3/ IRF4 signaling is required for $\mathrm{M} 2$ differentiation of $\mathrm{dM} \varphi$ induced by RANKL at the maternal-fetal interface in vitro and in vivo. Further studies should clarify the molecular mechanisms by which RANKL specifically activates AktSTAT6 signaling in $\mathrm{dM} \varphi$.

In human pregnancy, embryo implantation in the receptive endometrium triggers a series of responses collectively called decidualization. During decidualization, endometrial stromal cells (ESCs) undergo steroid hormone-dependent proliferation and differentiation into decidual cells. ${ }^{48}$ Interestingly, pregnancy-associated hormones (PAHs, such as estrogen and prolactin) upregulate RANKL and RANK levels, downregulate OPG expression, and further affect osteoclastogenesis at distinct stages of development. ${ }^{49,50}$ RANKL/RANK system also controls the incidence and onset of progestindriven breast cancer and physiological thermoregulation in females under the control of sex hormones. ${ }^{51,52}$ Therefore, high levels of PAH during pregnancy may also be one of the important factors leading to such high levels of RANKL/RANK at the maternal-fetal interface.

The decidua has been considered a specialized mucosal wall of the uterus. Research examining other mucosal tissues (skin and intestine) shows that epidermal and Peyer's patchderived DCs stimulated with RANKL induce immunosuppressive activity by modulating surface barrier DCs and increasing the expansion and function of Treg cells. ${ }^{26-28}$ Our results partially echo the immunosuppressive effect of RANKL in the mucosa. This function in the decidua is independent of the regulation of Treg differentiation.

In comparison with normal pregnancy, we observed that RANKL in trophoblasts and DSCs and RANK on $\mathrm{dM} \varphi$ in patients with miscarriage were greatly decreased. Moreover, the $\mathrm{dM} \varphi$ phenotype during human and mouse pregnancy wastage shows an $\mathrm{M} 1$ predominance. RANKL ${ }^{-/}$-mice presented $\mathrm{uM} \varphi$ dysfunction and increased fetal loss. This deregulation of $\mathrm{uM} \varphi$ supports an inflammatory environment that further triggers abortive processes. ${ }^{53}$ Therefore, our study reveals a novel pathogenic role of abnormal RANKL/RANK signaling at the maternal-fetal interface during SA in humans and mice. Trials conducted in vivo 

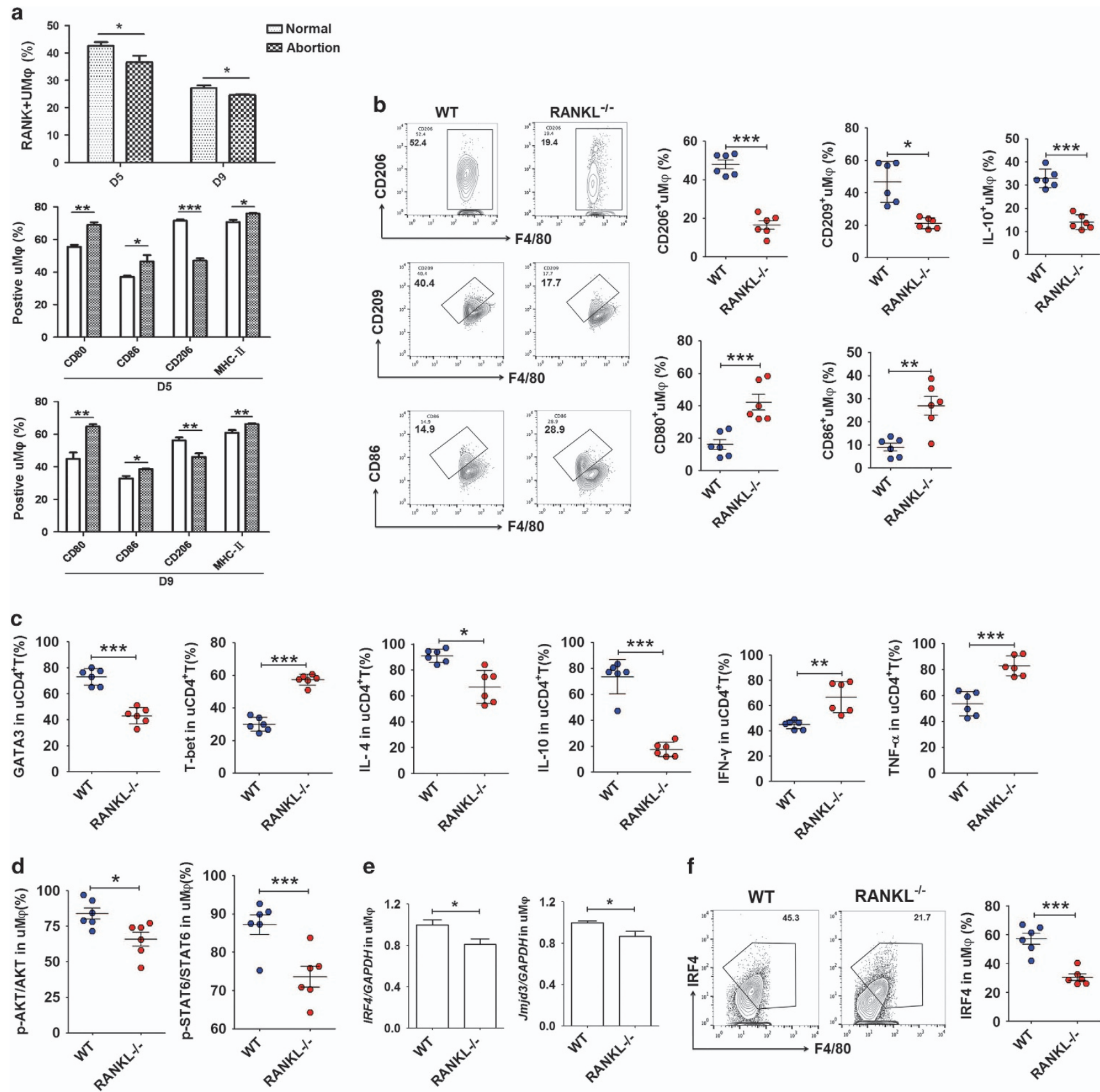

e
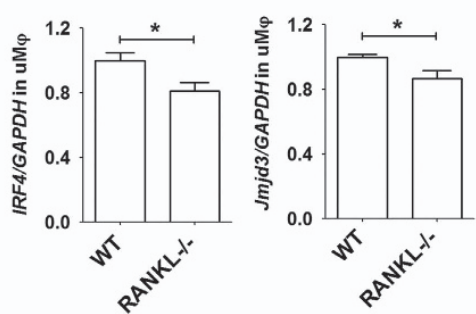

f
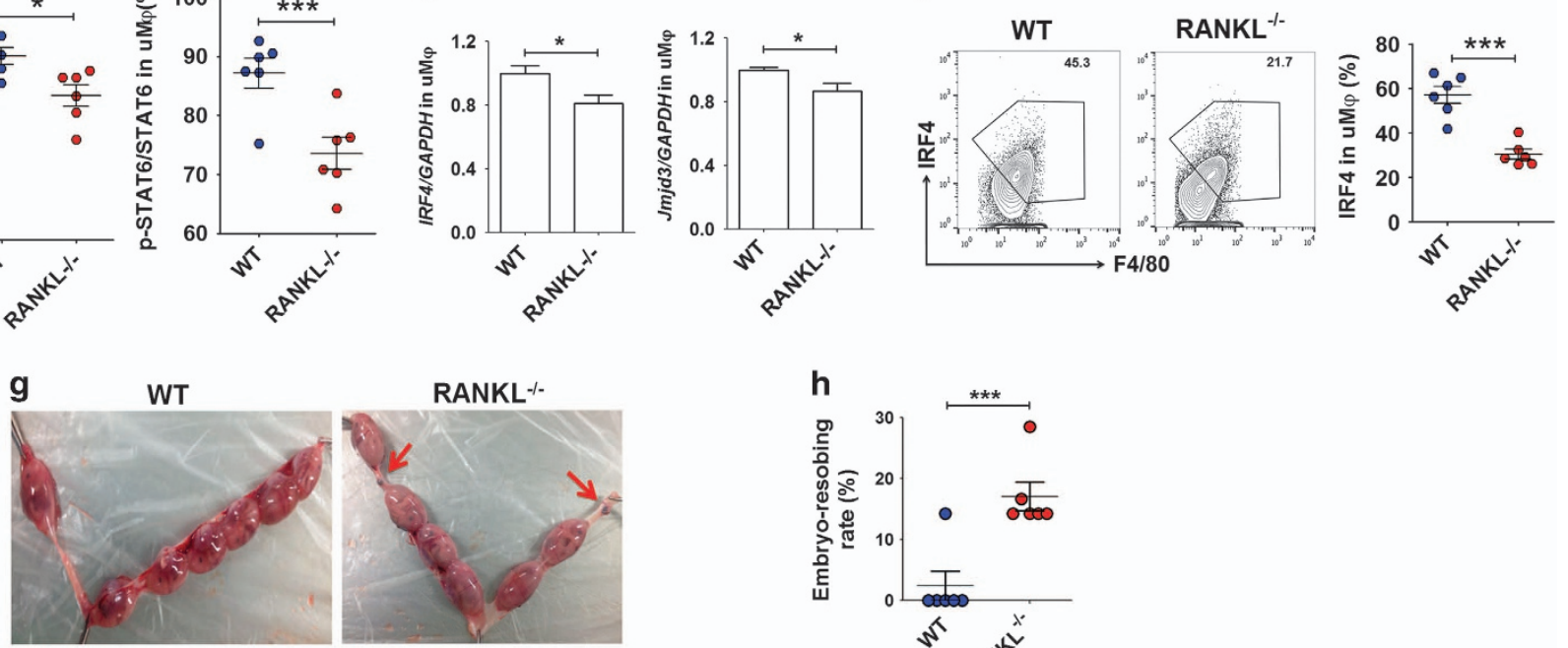

RANKL $^{-1}$
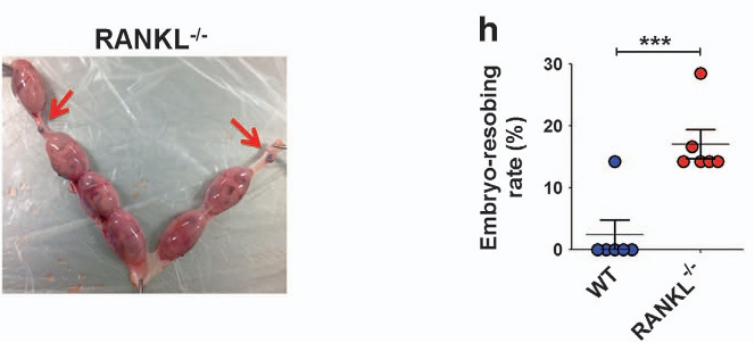

also showed that $\mathrm{RANKL}^{-/}$-mice had no significant influence on the total number of embryo implantations (data not shown). However, our unpublished data show that either endogenous or exogenous RANKL directly stimulates the proliferation and enhances the invasiveness of human trophoblasts, partially echoing its role in tumor cells. ${ }^{19}$ We propose that the lack of RANKL in vivo may result in a decrease in trophoblast proliferation and invasion, but to a certain extent, it will also 
Figure 4 Absence of RANKL expression leads to mouse uM $\varphi$ dysfunction and fetal loss. (a) RANK expression on uM $\varphi$ from CBA/Jo $\times$ DBA/2 ${ }^{\circ}$ matings (the abortion-prone model) and CBA/Jo $\times$ BALB/Cơ matings (normal pregnancy model) at days 5 and 9 of gestation ( $n=6$ mice per group). Moreover, the expression of CD80, CD86, CD206 and $\mathrm{MHCll}$ on $\mathrm{F} 4 / 80^{+} \mathrm{uM} \varphi$ from $\mathrm{CBA} / \mathrm{J} \rho \times \mathrm{DBA} / 2{ }^{*}$ matings (the abortion-prone model) and $\mathrm{CBA} / \mathrm{J} \rho \times \mathrm{BALB} / \mathrm{c}{ }^{-}$matings (normal pregnancy model) at days 5 and 9 of gestation ( $n=6$ mice per group); (adjusted t-test). (b) FCM analysis of CD206, CD209, IL-10, CD80 and CD86 in uM $\varphi$ of wild-type and RANKL knockout pregnant mice at day 10 ( $n=6$ mice per group); (Student's t-test). (c) FCM analysis of GATA-3, T-bet, IL-4, IL-10, IFN- $\gamma$ and TNF- $\alpha$ in uCD4 ${ }^{+}$T cells of WT and RANKL ${ }^{-1-}$ pregnant mice at day 10 ( $n=5$ mice per group); (Student's t-test). (d) FCM analysis of the phosphorylation level of Akt and STAT6 in uM $\varphi$ cells of WTand RANKL ${ }^{-1}$ - pregnant mice at day 10 ( $n=6$ mice per group); (Student's $t$-test). (e) uM $\varphi$ were isolated from mouse uterus ( $n=20$ mice per group) from WTand RANKL ${ }^{-1-}$ mice at day 10 of gestation by MACS, and then used to analyze the transcription of Jmid3 and IRF4 in uM $\varphi$. (Student's t-test). (f) FCM analysis of IRF4 levels in uM $\varphi$ cells of WT and RANKL ${ }^{-l-}$ pregnant mice at day 10 ( $n=6$ mice per group); (Student's $t$-test). ( $\mathbf{g}$ and $\mathbf{h}$ ) The embryo absorption rate in WTand RANKL ${ }^{-1}$ pregnant mice ( $n=6$ mice per group) was determined on day 14 of gestation. Fetal loss sites could be identified as hemorrhagic spots and necrosis (red arrows, left); (adjusted $t$-test). uM $\varphi$ : M $\varphi$ from mouse uterus; uCD4 ${ }^{+} T$ cells: $\mathrm{CD}_{4}^{+} \mathrm{T}$ cells from mouse uterus; Normal: normal pregnant mouse model; Abortion: abortion mouse model. D5: day 5 of gestation; D9: day 9 of gestation. WT: wild-type pregnant mice; RANKL ${ }^{-1-}:$ RANKL knockout pregnant $^{2}$ mice. Data are expressed as the mean \pm S.E.M. ${ }^{*} P<0.05,{ }^{\star *} P<0.01$ and ${ }^{\star \star *} P<0.001$

a

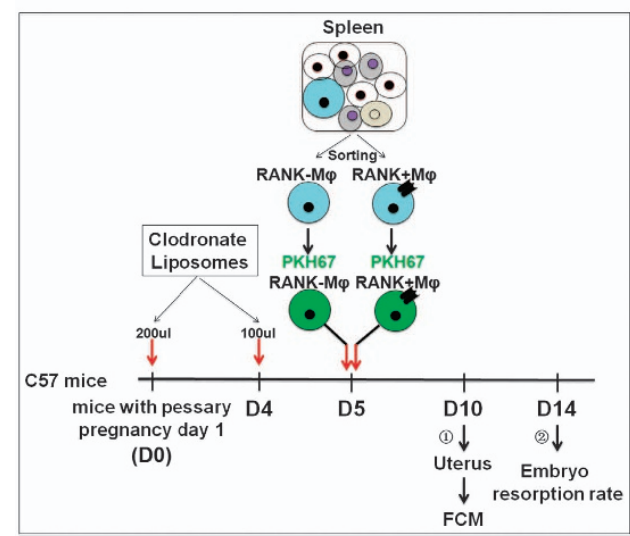

b
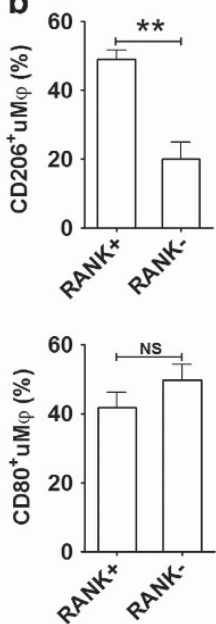
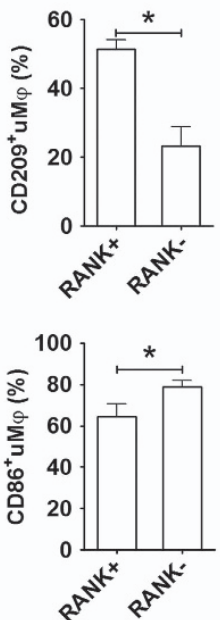

C

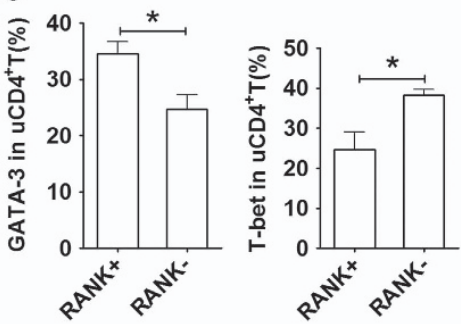

d

e
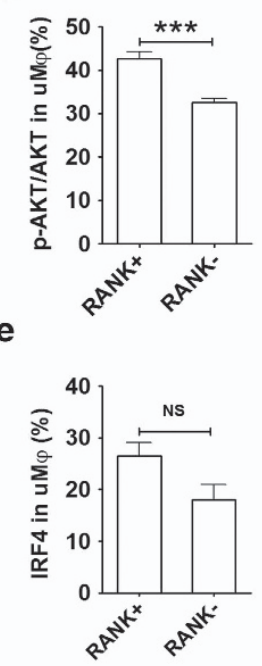
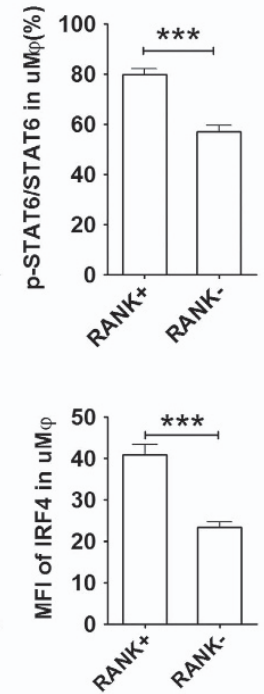

f

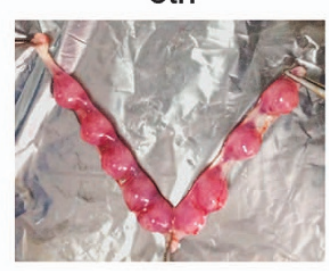

h RANK $+M \varphi$

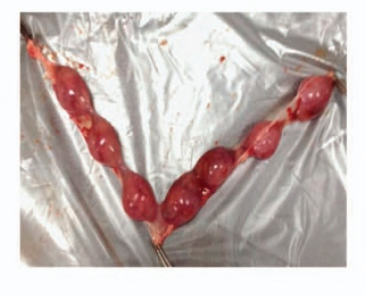

$M \varphi^{-1-}$

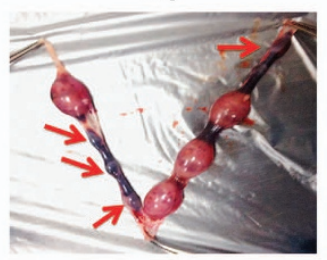

RANK-M $\varphi$

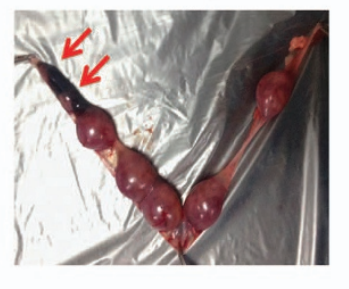

g

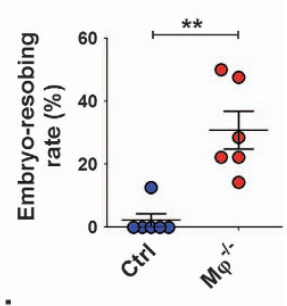

i

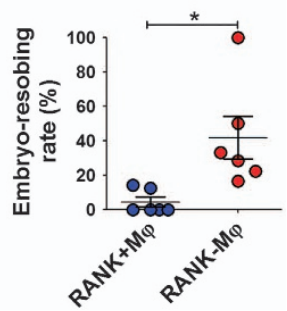

Figure 5 Adoptive transfer of RANK ${ }^{+} M \varphi$ relieves mouse embryo absorption induced by $M \varphi$ depletion. (a) RANK ${ }^{+}$and RANK ${ }^{-} \mathrm{M} \varphi s$ were isolated from mouse spleen, labeled with PKH-67, and then transferred to M $\varphi$-depleted pregnant mice at day 5 of gestation. The uterus was then collected and analyzed by FCM at day 10 , and the embryo resorption ratio was observed at day 14. (b) FCM analysis of CD206, CD209, CD80 and CD86 in PKH-67-uM $\varphi$ with PKH-67-RANK ${ }^{+} M \varphi$ and PKH-67-RANK ${ }^{-} \mathrm{M} \varphi$ transfer at day 10 ( $n=5$ mice per group). (Student's t-test). (c) FCM analysis of GATA-3 and T-bet in PKH-67-uM $\varphi$ with PKH-67-RANK $\mathrm{M} \varphi$ and PKH-67-RANK ${ }^{-} \mathrm{M} \varphi$ transfer at day 10 ( $n=5$ mice per group); (Student's t-test). (d) FCM analysis of the phosphorylation level of Akt and STAT6 in uM $\varphi$ cells with PKH-67-RANK ${ }^{+} M \varphi$ and PKH-67-RANK ${ }^{-} \mathrm{M} \varphi$ transfer at day 10 ( $n=5$ mice per group); (Student's t-test). (e) FCM analysis of the percentage and median fluorescence intensity (MFI) of IRF4 in uM $\varphi$ cells with PKH-67-RANK ${ }^{+} M \varphi$ and PKH-67-RANK ${ }^{-} \mathrm{M} \varphi$ transfer at day 10 ( $n=5$ mice per group); (Student's t-test). ( $\mathbf{f}$ and $\mathbf{g}$ ) The embryo absorption rate in ctrl pregnant C57BL/6 mice and pregnant C57BL/6 mice with $\mathrm{M} \varphi$ depletion ( $n=6$ mice per group) were counted on day 14 of gestation (adjusted $t$-test). ( $h$ and i) The embryo absorption rate in pregnant $\mathrm{C} 57 \mathrm{BL} / 6$ mice with $\mathrm{M} \varphi$ depletion ( $n=6$ mice per group) was counted on day 14 of gestation, after adoptive transfer of $\operatorname{RANK}{ }^{+} \mathrm{M} \varphi$ and $\mathrm{RANK}^{-} \mathrm{M} \varphi$ at day 5 (adjusted $t$-test). RANK : adoptive transfer of PKH-67$\mathrm{RANK}^{+} \mathrm{M} \varphi$; $\mathrm{RANK}^{-}$: adoptive transfer of PKH-67-RANK ${ }^{-} \mathrm{M} \varphi$; uM $\varphi$ : M $\varphi$ from mouse uterus. Data are expressed as the mean $\pm \mathrm{S} . \mathrm{E} \cdot \mathrm{M} .{ }^{*} P<0.05,{ }^{* *} P<0.01$ and ${ }^{* * *} P<0.001$ 
a
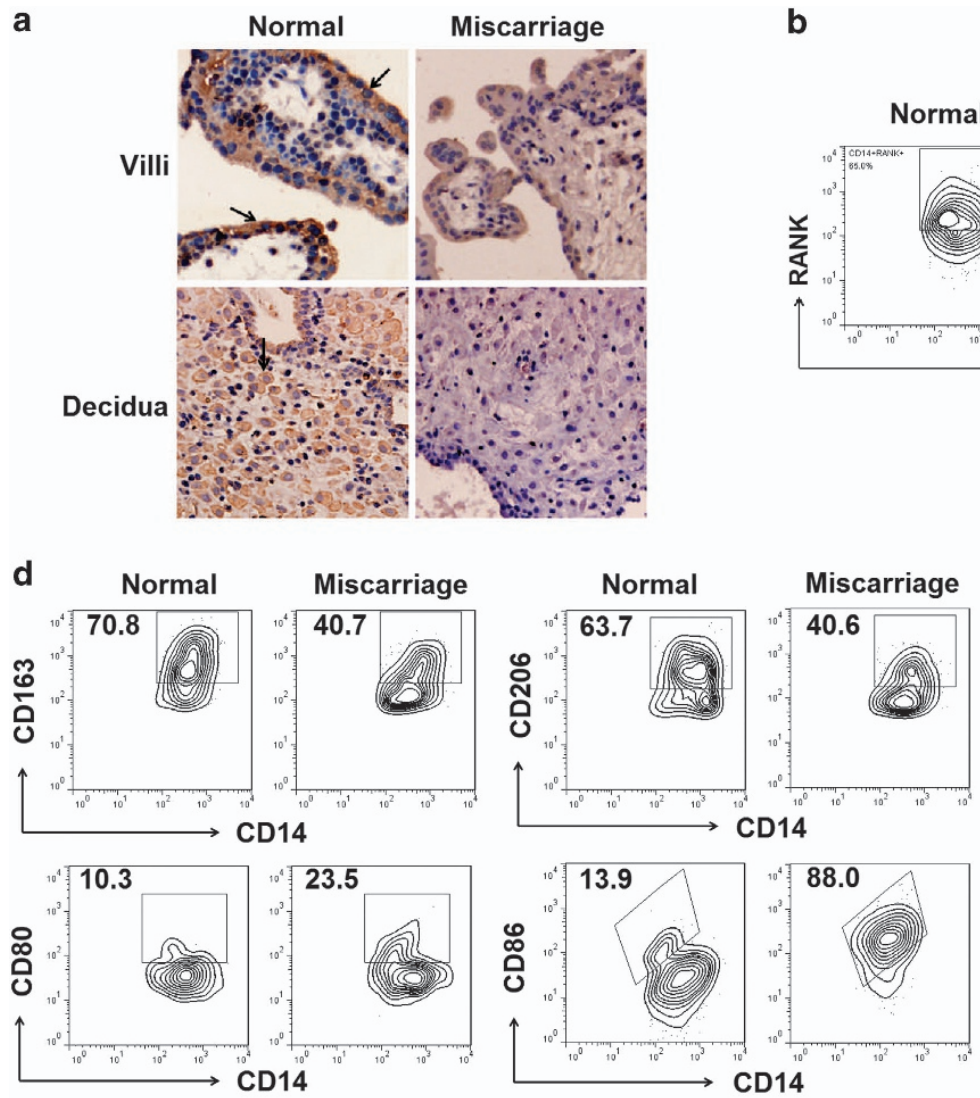

b

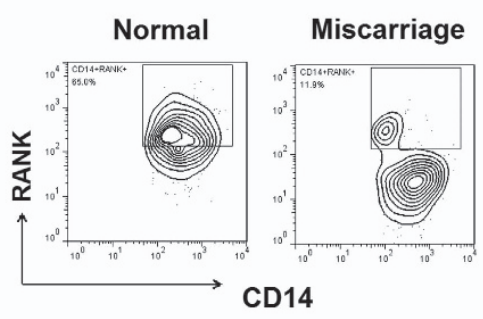

e
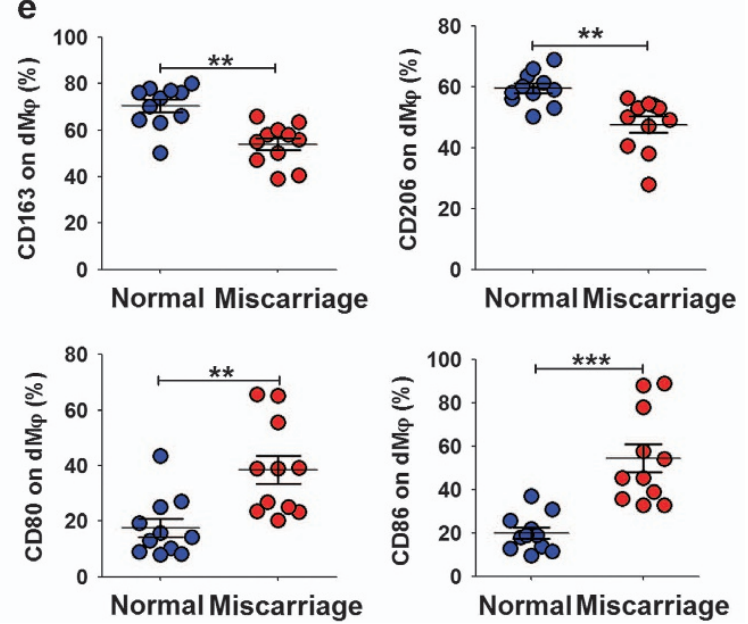

C

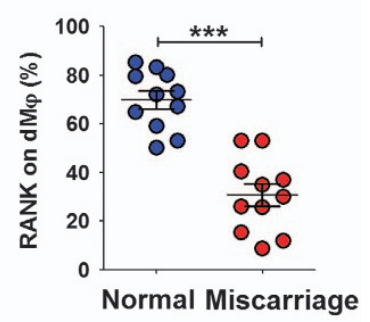

Figure 6 There are low levels of RANKL/RANK at the maternal-fetal interface during miscarriage. (a) Immunohistochemistry analysis of RANKL expression in villi and deciduas from women with normal pregnancy $(n=12)$ or miscarriage $(n=12)$ during the first trimester. RANKL expression was localized to the cell membrane and the cytoplasm (arrows) in the deciduas and villi. Original magnification: $\times 200$. ( $\mathbf{b}$ and $\mathbf{c})$ FCM analysis of the percentage of RANK ${ }^{+} \mathrm{dM} \varphi$ from women with normal pregnancy $(n=11)$ or miscarriage $(n=11)$ during the first-trimester. (d and e) $\mathrm{FCM}$ analysis of the percentage of $\mathrm{CD}_{163^{+}}, \mathrm{CD}_{20}{ }^{+}, \mathrm{CD} 80^{+}$and $\mathrm{CD} 86^{+} \mathrm{dM} \varphi$ from women with normal pregnancy $(n=11)$ or miscarriage $(n=11)$ during the first trimester. Normal: normal pregnant women; Miscarriage: SA women. Data are expressed as the mean \pm S.E.M. ${ }^{*} P<0.01$ and ${ }^{* * *} P<0.001$ (Student's t-test)

create a proinflammatory microenvironment. This inflammatory pattern during the initial stage of pregnancy may be conducive to the invasion and implantation of trophoblasts. Therefore, the overall effect of absent RANKL signaling in vivo may not affect embryo implantation. However, with advancing pregnancy, abnormally low levels of RANKL will result in miscarriage via the $\mathrm{M} 1 \mathrm{dM} \varphi$-triggered disorder of maternal-fetal immune tolerance. Therefore, further research is needed to elucidate the cause of low RANKL/RANK expression in miscarriage patients.

In conclusion, as shown in Figure 7, accompanied by the implantation of blastocyst during normal pregnancy, $\mathrm{PAH}$ trigger ESCs to differentiate into DSCs and further induce high levels of RANKL expression and CCL2 release. The latter allows the recruitment of peripheral monocytes into decidua. Embryonic trophoblasts that are deeply implanted in decidua are in close contact with DSCs and DLCs. The dialog of these cells not only further increases RANKL expression in trophoblasts and DSCs but also enhances the sensitivity of RANK on $\mathrm{dM} \varphi$ to RANKL by upregulating RANK expression. Subsequently, the RANKL-RANK interaction drives $\mathrm{dM} \varphi$ to $\mathrm{M} 2$ differentiation. After being instructed, these $\mathrm{dM} \varphi$ will create and maintain a maternal-fetal tolerant microenvironment.
Once the maternal-fetal interface presents abnormal low level of RANKL, dysfunction of $\mathrm{dM} \varphi$ and then miscarriage will occur. Therefore, our study provides a potential target molecule, RANKL, for the identification of new strategies to prevent and treat pregnancy complications.

\section{Materials and Methods}

Patient and sample collection. All procedures involving participants in this study were approved by the Human Research Ethics Committee of Obstetrics and Gynecology Hospital, Fudan University (Shanghai, China), and all subjects completed an informed consent to collect tissue samples. First-trimester human peripheral blood was obtained from 41 women with clinically normal pregnancies (age: $27.45 \pm 7.21$ years; gestational age at sampling: $48.35 \pm 7.6$ days (mean $\pm \mathrm{S}$. D.)]), which were terminated for nonmedical reasons. Human villi tissues were obtained from 172 women with clinically normal pregnancies (age: $29.88 \pm 6.91$ years; gestational age at sampling: $49.17 \pm 9.34$ days [mean \pm S.D.]) (Termination for nonmedical reasons), or from 12 women with SA (age: $31.09 \pm 4.28$ years; gestational age at sampling: $47.95 \pm 10.1$ days (mean \pm S.D.)). Decidual tissues were obtained from 135 women with clinically normal pregnancies (age: $27.19 \pm 5.61$ years; gestational age at sampling: $51.09 \pm 8.72$ days (mean \pm S.D.)) (termination for nonmedical reasons) and 23 women with spontaneous miscarriage (age: $31.54 \pm 5.71$ years; gestational age at sampling: $53.06 \pm 5.8$ days (mean \pm S.D.)). All pregnant women were confirmed by ultrasound and blood tests, and the women with spontaneous miscarriage because of endocrine, anatomical, and genetic abnormalities, as well as infection were excluded. 


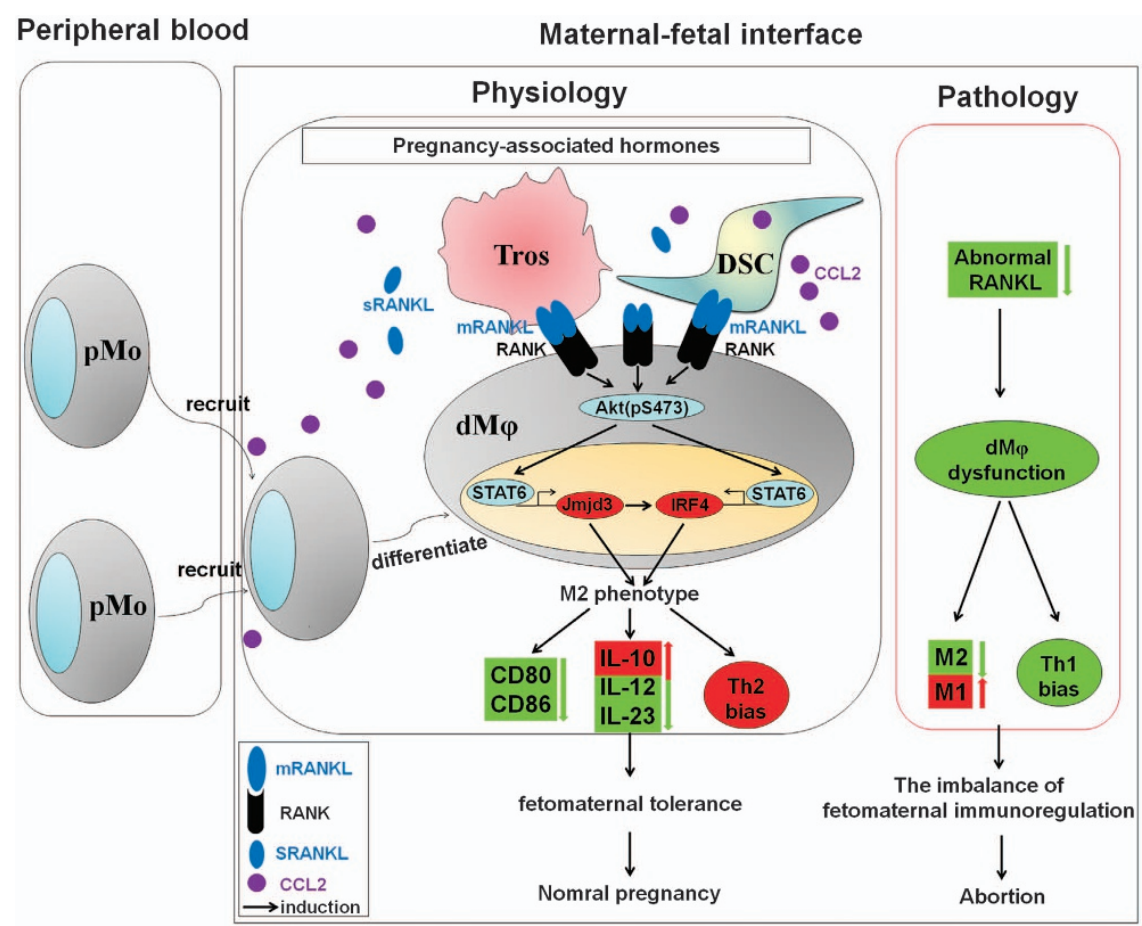

Figure 7 RANKL-mediated harmonious dialog between the fetus and mother guarantees a smooth gestation by inducing decidual M2 macrophage polarization. Together with blastocyst implantation during normal pregnancy, PAHs trigger ESCs to differentiate into DSCs, further inducing high levels of RANKL expression and CCL2 release. The latter recruits more peripheral monocytes to the decidua. Embryonic trophoblasts that are deeply implanted in decidua can closely contact DSCs and DLCs. This dialog not only further increases RANKL expression in trophoblasts and DSCs, but it also enhances the sensitivity of RANK to RANKL by upregulating RANK expression on M $\varphi$. Subsequently, the RANKL-RANK interaction drives M $\varphi$ to M2 differentiation (low expression of CD80 and CD86, high secretion of IL-10, and low level of IL-12 and IL-23) by activating Akt/ STAT6-Jmjd3/IRF4 signaling. After education, these $M \varphi s$ will create and maintain a maternal immune tolerance microenvironment (increase in Th2 and decrease in Th1). After the development of abnormally low RANKL expression at the maternal-fetal interface, the dysfunction of $\mathrm{M} \varphi$, the imbalance of maternal-fetal immune regulation and then abortion will occur. Tro: trophoblasts

Cell line. The human placental choriocarcinoma cell line (JEG-3 cells) was purchased from Bank of Cell, Chinese Academy of Sciences, Shanghai, China.

Mice. RANKL heterozygote mice were obtained from the Jackson Laboratories (Sacramento, CA, USA) and subsequently maintained in the Laboratory Animal Facility of Fudan University (Shanghai, China). RANKL knockout (RANKL ${ }^{-1}$ ) mice and wildtype littermates (WT) were obtained by mating of male and female RANKL heterozygote mice. A group of adult female C57BL/6 mice were purchased from the Laboratory Animal Facility of Fudan University and used for this study. They were usually maintained for 2 weeks in the animal facility before use. The Animal Care and Use Committee of Obstetrics and Gynecology Hospital, Fudan University approved all animal protocols.

Immunohistochemistry. Immunohistological staining was performed as previously described. ${ }^{46}$ Human villi and deciduas were labeled with mouse antiRANKL Abs (15 $\mu \mathrm{g} / \mathrm{ml}$, MAB626, R\&D Systems, Abingdon, UK).

Antibodies for FCM. For the identification of cell purity, primary trophoblasts and DSCs were stained with phycoerythrin (PE)-conjugated anti-human vimentin (562337; BD Biosciences) and fluorescein isothiocyanate (FITC)-conjugated antihuman cytokeratin (347653; BD Biosciences, San Jose, CA, USA); DLCs were stained with allophycocyanin (APC)-conjugated anti-human CD45 antibody (304012; Biolegend); $\mathrm{dM} \varphi$ was stained with FITC-conjugated anti-human CD14 antibody (301804; Biolegend); decidual naive T cells were stained with FITC-conjugated antihuman CD4 antibody (300506; Biolegend) and PE-conjugated anti-human CD45RA antibody (304107; Biolegend, San Diego, CA, USA).

PE-conjugated anti-human RANKL antibody (347504; Biolegend) and PEconjugated anti-human RANK antibody (FAB683P; R\&D Systems) were used to analyze the expression of RANKL on trophoblasts and DSCs, and the expression of RANK on $\mathrm{dM} \varphi$; pMo and or $\mathrm{dM} \varphi$ were stained with FITC-conjugated anti-human CD14 antibody (301804), phycoerythrin -cyanine 7 (PE-Cy7)-conjugated CD80 antibody (305218), APC-conjugated CD86 antibody (305412), PE-Cy7-conjugated IL-10 antibody (501420), PE-conjugated IL-12/23p40 antibody (501807), APCconjugated IFN- $\gamma$ (502511), APC-conjugated CD206 (321110), allophycocyanincyanine 5.5 (PerCP/Cy5.5)-conjugated CD209 antibody (330110) and PE-conjugated CD163 antibody (333606) (all from Biolegend). Decidual naive T cells were stained with PE-Cy7-conjugated CD4 antibody (303718), Alexa Fluor 488-conjugated antiGATA-3 (653808) and PE-conjugated anti-T-bet (644810) (all from Biolegend).

The uSC and pTros were stained with PE anti-mouse RANKL antibody (510005); the $\mathrm{uM} \varphi$ was stained with PE anti-mouse RANK antibody (119805), Alexa Fluor 647 antimouse F4/80 antibody (122610), PE/Cy7 anti-mouse CD45 antibody (103114), FITC anti-mouse CD11b antibody (101206), FITC anti-mouse CD80 antibody (104706), PE anti-mouse CD86 antibody (105008), FITC anti-mouse CD206 (MMR) antibody (141704), Brilliant Violet 421 (BV421) anti-mouse IL-10 antibody (505021) (all from Biolegend), PE anti-mouse CD209 (DC-SIGN) antibody (12-2091; ebioscience; Thermo Fisher Scientific, Inc., Waltham, MA, USA), PE anti-mouse MHCll antibody (12-5322; ebioscience), and PE/Cy7 anti-IRF4 antibody (25-9858; ebioscience); UCD4 ${ }^{+} T$ cells were stained with PE/Cy7 anti-mouse CD45 antibody (103114), FITC anti-mouse CD4 antibody (100405), PE anti-mouse IL-4 antibody (504103), BV421 anti-mouse IL-10 antibody (505021), APC anti-mouse IFN- $\gamma$ antibody (505810), PE anti-mouse TNF- $\alpha$ antibody (506305) (all from Biolegend), BV421 anti-mouse GATA-3 antibody (563349; BD Biosciences), and PE anti-mouse T-bet antibody (561268; BD Biosciences).

For intracellular staining of signaling molecules in $\mathrm{dM} \varphi$ or $\mathrm{uM} \varphi$, cells were permeabilized (Cytofix/Cytoperm kit; BD Biosciences) and incubated with Alexa Fluor 647-conjugated Akt (pS473) antibody (561670) and Alexa Fluor 488-conjugated Stat6 (pY641) antibody (612600 for dM $\varphi$; 558243 for $\mathrm{uM} \varphi$ ) (Phosflow antibodies were from $\mathrm{BD}$ Biosciences) .

Isolation and culture of human trophoblasts, DSCs and DLCs. The villi and decidua tissues from the first-trimester pregnancy were put immediately into ice-cold Dulbecco's modified Eagle's medium (DMEM high D-glucose; Gibco, Grand Island, NY, USA), transported to the laboratory within 30 min after surgery 
and washed in Hank's balanced salt solution for isolation of human trophoblasts from villi, and DSCs and DLCs from deciduas according to a previously described method. ${ }^{46}$ This method supplies a $95 \%$ purity of vimentin ${ }^{-}$cytokeratin $(\mathrm{CK}) 7^{+}$ trophoblast cells and greater than $98 \%$ vimentin ${ }^{+} \mathrm{CK} 7^{-}$DSCs and $\mathrm{CD} 45^{+} \mathrm{DLCs}$, which was confirmed by FCM analysis.

Enzyme-linked immunosorbent assay (ELISA). Cytokine concentrations were measured using ELISA kits (R\&D Systems).

Detection of RANK expression on peripheral blood mononuclear cells (PBMCs) and DLCs. The PBMCs were isolated from the peripheral blood of pregnant women who were terminated for nonmedical reasons. Next, FCM was performed to analyze the expression of RANK on pMo and $\mathrm{dM} \varphi$ by labeling anti-human CD14, RANK and CD45 antibodies. In addition, we further evaluated the phenotype of RANK $^{+}$and RANK ${ }^{-}$pMos and $\mathrm{dM} \varphi$ purified from PBMC and DLC $(n=24)$ by FCM.

Purification of $\operatorname{dM} \varphi$ and decidual naive T cells. We isolated $\mathrm{dM} \varphi$ and decidual naive T cells from DLCs by MACS (Miltenyi Biotec, Bergisch Gladbach, Germany) and performed FCM analysis with standard protocols.

RANKL-overexpressed JEG-3 cells and DSCs. We obtained RANKLoverexpressed JEG-3 cells and DSCs by transfection with pcDNA(+)-RANKL plasmid, and the results were confirmed by FCM analysis. The pcDNA(+)-RANKL plasmid and pcDNA(+)-vector plasmid were from GeneChem Co., Ltd (Shanghai, China).

Co-culture of trophoblasts/JEG-3 cells, DSCs and $\mathrm{dM} \varphi$. The $\mathrm{dM} \varphi$ were cultured in culture medium, directly contacted with primary trophoblasts/JEG-3 cells and or DSCs at a $1: 1: 1$ ratio. In addition, $5 \mu \mathrm{g} / \mathrm{ml}$ anti-human RANKL neutralizing antibody (AB626, R\&D Systems), $100 \mathrm{ng} / \mathrm{ml}$ rhOPG protein (185OS-025, R\&D Systems), $10 \mu \mathrm{M}$ LY294002 (Cells Signal Technology, Danvers, MA, USA) or $21 \mathrm{nM}$ STAT6 signal inhibitor (STAT6i) AS1517499 (Axon Medchem, Groningen, The Netherlands) was added to the co-culture unit. After $48 \mathrm{~h}$, the expression of RANKL on trophoblasts and DSCs, the expression of RANK, and the M1 phenotype and M2 phenotype on $\mathrm{dM} \varphi$ were analyzed by $\mathrm{FCM}$, and the concentration of IL-10, IL-12p40 and IL-23 in the supernatants was detected by ELISA (R\&D Systems).

The intracellular phosphorylation level of Akt and STAT6. The intracellular phosphorylation level of Akt and STAT6 in $\mathrm{dM} \varphi$ after 24-h culture with JEG-3 and DSCs was analyzed using BD Phosflow antibodies, according to standard protocols.

The transcription of Jmjd3, IRF4 and IRF5. After 24-h co-culture, the transcription level of Jmid3, IRF4 and IRF5 in $\mathrm{dM} \varphi$ was analyzed by real-time PCR, according to standard protocols. The primer sequences were designed and synthesized by TaKaRa Biotechnology Co., Ltd (Tokyo, Japan) as described in Supplementary Table 1.

Co-culture of $\mathrm{dM} \varphi$ and decidual naive T cells. After $48 \mathrm{~h}$ of culture with trophoblasts/JEG-3 cells and DSCs, CD14 $4^{+} \mathrm{dM} \varphi$ were collected and washed three times with phosphate-buffered saline to remove excess cytokines. The remaining cells were co-cultured with autologous decidual naive $T$ cells at a $1: 1$ ratio. The decidual naive T cells were pre-activated with $5 \mu \mathrm{g} / \mathrm{ml}$ anti-CD3 (OKT3; 16-0037; ebioscience), $1 \mu \mathrm{g} / \mathrm{ml}$ anti-CD28 (CD28.2; 16-0289; ebioscience), and $20 \mathrm{U} / \mathrm{ml}$ rhlL-2 (202-IL-010, R\&D Systems) for 2 days, and collected, washed, and then incubated with culture medium only. After 5 days of co-culture, the naive $T$ cells were reactivated with anti-CD3 and anti-CD28 for $24 \mathrm{~h}$ before the supernatants were collected. The expression of GATA-3 and T-bet in decidual naive T cells, and the secretion level of IL-4, IL-10, and TNF- $\alpha$ and IFN- $\gamma$ were analyzed by FCM and ELISA (R\&D Systems), respectively.

Animals and experimental design. We divided female C57BL/6 mice (age: 8 weeks old, weight: 20-23 g) into two groups by using a random number table by body weight, age and family: the adoptive transfer of RANK ${ }^{+} \mathrm{M} \varphi$ group and the adoptive transfer of RANK ${ }^{-} \mathrm{M} \varphi$ group. This was an unblinded trial.

The differentiation of $\mathrm{uM} \varphi$ and $\mathrm{uCD} 4^{+} \mathrm{T}$ cells, the activation of Akt and STAT6, and the level of IRF4 in uM $\varphi$ were analyzed by FCM, and the level of fetal loss was counted at day 14 of gestation in WT and RANKL ${ }^{-/-}$pregnant mice. The day of appearance of a copulatory plug was arbitrarily designated as day 0 of gestation. The embryo absorption rate and implantation number were counted on day 14 of gestation. The percentage of fetal loss (the embryo absorption rate) was calculated as follows: percentage fetal loss $=R /(R+V) \times 100$, where $R$ represents the number of hemorrhagic implantatio (sites of fetal loss) and $V$ stands for the number of viable, surviving fetuses.

In addition, mouse uterine tissues were removed, minced on ice and digested with an enzyme mix of Liberase and Dispase (Invitrogen, Carlsbad, CA, USA). UM $\varphi$ were isolated from mouse uterus by MACS (Miltenyi Biotec) to analyze the transcription of Jmid3 and IRF4 (Takara Bio Inc.,Tokyo, Japan) by RT-PCR at day 10 of gestation. The primer sequences were described in Supplementary Table 1.

For $\mathrm{M} \varphi$ depletion and $\mathrm{M} \varphi$ adoptive transfer in pregnant $\mathrm{C} 57 \mathrm{BL} / 6$ mice, and Clodronate Liposomes were injected intraperitoneally at day $1(200 \mu \mathrm{l})$ and day 4 $(100 \mu l)$ of gestation, and then the expression of RANKL in Vimentin ${ }^{+}$USC and $C K 7^{+}$ pTros were analyzed at day 7 by FCM. RANK ${ }^{+}$and $\mathrm{RANK}^{-} \mathrm{M} \varphi$ s were isolated from mouse spleen and labeled with PKH-67, and then they were transferred to $M \varphi$ depleted pregnant mice at day 5 of gestation. Subsequently, the differentiation of $\mathrm{uM} \varphi$ and $\mathrm{UCD}^{+}{ }^{+} \mathrm{T}$ cells, the activation of Akt and STAT6, and the IRF4 level in $\mathrm{uM} \varphi$ were analyzed by FCM at day 10 of gestation, and the level of fetal loss was counted at day 14 of gestation in the RANK ${ }^{+}$transfer group and RANK ${ }^{-}$transfer group.

To investigate the role of STAT6 and Jmjd3 signals in $\mathrm{UM} \varphi$ and $\mathrm{uCD} 4^{+} \mathrm{T}$ cells, the pregnant C57BL/6 mice were intraperitoneally injected with STAT6i AS1517499 (200 $\mu$ lat the concentration of $2 \mathrm{mg} / \mathrm{kg})$ or JMJD3i GSK J4 HCl $(200 \mu \mathrm{l}$ at a concentration of $4.48 \mathrm{mg} / \mathrm{kg}$ ) in ( $n=5$ mice per group) at day 4 , and then the differentiation of $\mathrm{uM} \varphi$ and $\mathrm{u}$ $\mathrm{CD}^{+} \mathrm{T}$ cells and the IRF4 level in uM $\varphi$ cells at day 10 were determined by FCM.

Statistics. The continuous variable is shown as the mean \pm S.E.M. Continuous variables were analyzed by Student's t-test in case of two groups and by one-way ANOVA using Tukey's post-hoc test in multiple groups. The embryo resorbing rate was analyzed using an adjusted $t$-test. All analyses were conducted with SPSS 16.0 Statistical Package for the Social Sciences software (IBM SPSS, Armonk, NY, USA). $P<0.05$ was considered statistically significant.

\section{Conflict of Interest}

The authors declare no conflict of interest.

Acknowledgements. We thank Professor Petra Clara Arck from Hamburg University (Hamburg, Germany) for her kind suggestions regarding the design of this study and language editing of the manuscript. We thank Assistant Professor Yi-Qin Wang from the Department of Pathology, Hospital of Obstetrics and Gynecology, Fudan University for helping with the histological analysis. This study was supported by the National Basic Research Program of China (2015CB943300), and Major Program of National Natural Science Foundation of China (NSFC) (31671200, 81490744, 91542108, 81471513, 81571275 and 81671460), the Oriented Project of Science and Technology Innovation from Key Lab. of Reproduction Regulation of NPFPC (CX2017-2), the Shanghai Rising-Star Program 16QA1400800, the Training Program for young talents of Shanghai Health System XYQ2013104 and the Program for Zhuoxue of Fudan University, the Program for Shanghai leaders, the Program of Shanghai Outstanding Academic Leader (15XD1500900), and the Postdoctoral Science Foundation of China No.2014M550573.

\section{Author contributions}

Y-HM and W-JZ conducted all experiments and prepared the figures and the manuscript. L-PJ and JW edited the manuscript. L-BL and K-KC assisted with the flow cytometry assay and animal trials. $\mathrm{HL}$ assisted with the sample collection. JM assisted with the IHC assay. M-QL and D-JL designed the research, supervised the project and edited the manuscript. All authors were involved in writing the manuscript.

\section{Publisher's Note}

Springer Nature remains neutral with regard to jurisdictional claims in published maps and institutional affiliations.

1. Trowsdale J, Betz AG. Mother's little helpers: mechanisms of maternal-fetal tolerance. Nat Immunol 2006; 7: 241-246.

2. Chaouat G, Ledee-Bataille N, Chea KB, Dubanchet S. Cytokines and implantation. Chem Immunol Allergy 2005; 88: 34-63.

3. Arck PC, Hecher K. Fetomaternal immune cross-talk and its consequences for maternal and offspring's health. Nat Med 2013; 19: 548-556.

4. Erlebacher A. Immunology of the maternal-fetal interface. Annu Rev Immunol 2013; 31: 387-411.

5. Redman CW, Sargent IL. Immunology of pre-eclampsia. Am J Reprod Immunol 2010; 63: 534-543. 
6. Svensson J, Jenmalm MC, Matussek A, Geffers R, Berg G, Ernerudh J. Macrophages at the fetal-maternal interface express markers of alternative activation and are induced by M-CSF and IL-10. J Immunol 2011; 187: 3671-3682.

7. Nagamatsu T, Schust DJ. The immunomodulatory roles of macrophages at the maternalfetal interface. Reprod Sci 2010; 17: 209-218.

8. Gordon S, Taylor PR. Monocyte and macrophage heterogeneity. Nat Rev Immunol 2005; 5 953-964.

9. Mantovani A, Sozzani S, Locati M, Allavena P, Sica A. Macrophage polarization: tumorassociated macrophages as a paradigm for polarized M2 mononuclear phagocytes. Trends Immunol 2002; 23: 549-555.

10. Biswas SK, Mantovani A. Macrophage plasticity and interaction with lymphocyte subsets: cancer as a paradigm. Nat Immunol 2010; 11: 889-896.

11. Gustafsson C, Mjösberg J, Matussek A, Geffers R, Matthiesen L, Berg G et al. Gene expression profiling of human decidual macrophages: evidence for immunosuppressive phenotype. PLOS ONE 2008; 3: e2078.

12. Houser BL, Tilburgs T, Hill J, Nicotra ML, Strominger JL. Two unique human decidual macrophage populations. J Immunol 2011; 186: 2633-2642.

13. Lacey DL, Timms E, Tan HL, Kelley MJ, Dunstan CR, Burgess T et al. Osteoprotegerin ligand is a cytokine that regulates osteoclast differentiation and activation. Cell 1998; 93: 165-176.

14. Yasuda H, Shima N, Nakagawa N, Yamaguchi K, Kinosaki M, Mochizuki S et al. Osteoclast differentiation factor is a ligand for osteoprotegerin/osteoclastogenesis-inhibitory factor and is identical to TRANCE/RANKL. Proc Natl Acad Sci USA 1998; 95: 3597-3602.

15. Kong YY, Yoshida H, Sarosi I, Tan HL, Timms E, Capparelli $\mathrm{C}$ et al. OPGL is a key regulator of osteoclastogenesis, lymphocyte development and lymph-node organogenesis. Nature 1999; 397: 315-323.

16. Rossi SW, Kim MY, Leibbrandt A, Parnell SM, Jenkinson WE, Glanville SH et al. RANK signals from $\mathrm{CD} 4(+) 3(-)$ inducer cells regulate development of Aire-expressing epithelial cells in the thymic medulla. J Exp Med 2007; 204: 1267-1272.

17. Fata JE, Kong YY, Li J, Sasaki T, Irie-Sasaki J, Moorehead RA et al. The osteoclast differentiation factor osteoprotegerin-ligand is essential for mammary gland development. Cell 2000; 103: 41-50.

18. Cao Y, Bonizzi G, Seagroves TN, Greten FR, Johnson R, Schmidt EV et al. IKKalpha provides an essential link between RANK signaling and cyclin D1 expression during mammary gland development. Cell 2001; 107: 763-775.

19. Jones DH, Nakashima T, Sanchez OH, Kozieradzki I, Komarova SV, Sarosi I et al. Regulation of cancer cell migration and bone metastasis by RANKL. Nature 2006; 440: 692-696.

20. Nakamura ES, Koizumi K, Kobayashi M, Saitoh Y, Arita Y, Nakayama T et al. RANKLinduced CCL22/macrophage-derived chemokine produced from osteoclasts potentially promotes the bone metastasis of lung cancer expressing its receptor CCR4. Clin Exp Metastasis 2006; 23: 9-18.

21. Kim MS, Day CJ, Morrison NA. MCP-1 is induced by receptor activator of nuclear factor\{kappa\}B ligand, promotes human osteoclast fusion, and rescues granulocyte macrophage colony-stimulating factor suppression of osteoclast formation. J Biol Chem 2005; 280: 16163-16169.

22. Meng YH, Li H, Chen X, Liu LB, Shao J, Chang KK et al. RANKL promotes the growth of decidual stromal cells in an autocrine manner via CCL2/CCR2 interaction in human early pregnancy. Placenta 2013; 34: 663-671.

23. Anderson DM, Maraskovsky E, Billingsley WL, Dougall WC, Tometsko ME, Roux ER et al. A homologue of the TNF receptor and its ligand enhance T-cell growth and dendritic-cell function. Nature 1997; 390: 175-179.

24. Wong BR, Josien R, Lee SY, Sauter B, Li HL, Steinman RM et al. TRANCE (tumor necrosis factor [TNF]-related activation-induced cytokine), a new TNF family member predominantly expressed in T cells, is a dendritic cell-specific survival factor. J Exp Med 1997; 186: 2075-2080.

25. Seshasayee D, Wang H, Lee WP, Gribling P, Ross J, Van Bruggen $\mathrm{N}$ et al. A novel in vivo role for osteoprotegerin ligand in activation of monocyte effector function and inflammatory response. J Biol Chem 2004; 279: 30202-30209.

26. Loser K, Mehling A, Loeser S, Apelt J, Kuhn A, Grabbe S et al. Epidermal RANKL controls regulatory T-cell numbers via activation of dendritic cells. Nat Med 2006; 12: 1372-1379.

27. Soontrapa K, Honda T, Sakata D, Yao C, Hirata T, Hori S et al. Prostaglandin E2prostaglandin E receptor subtype 4 (EP4) signaling mediates UV irradiation-induced systemic immunosuppression. Proc Natl Acad Sci USA 2011; 108: 6668-6673.

28. Totsuka T, Kanai T, Nemoto $\mathrm{Y}$, Tomita $\mathrm{T}$, Okamoto R, Tsuchiya $\mathrm{K}$ et al. RANK-RANKL signaling pathway is critically involved in the function of $\mathrm{CD} 4+\mathrm{CD} 25+$ regulatory $\mathrm{T}$ cells in chronic colitis. J Immunol 2009; 182: 6079-6087.

29. Boyle WJ, Simonet WS, Lacey DL. Osteoclast differentiation and activation. Nature 2003; 423: $337-342$

30. Mizukami J, Takaesu G, Akatsuka H, Sakurai H, Ninomiya-Tsuji J, Matsumoto K et al. Receptor activator of NF-kappaB ligand (RANKL) activates TAK1 mitogen-activated protein kinase kinase kinase through a signaling complex containing RANK, TAB2, and TRAF6. Mol Cell Biol 2002; 22: 992-1000.

31. Taniguchi R, Fukushima H, Osawa K, Maruyama T, Yasuda H, Weih F et al. RelB-induced expression of Cot, an MAP3K family member, rescues RANKL-induced osteoclastogenesis in alymphoplasia mice by promoting NF-kappaB2 processing by IKKalpha. J Biol Chem 2014; 289: 7349-7361.

32. Guerrini MM, Takayanagi $\mathrm{H}$. The immune system, bone and RANKL. Arch Biochem Biophys 2014; 561: 118-123.

33. Welch JS, Escoubet-Lozach L, Sykes DB, Liddiard K, Greaves DR, Glass CK. TH2 cytokines and allergic challenge induce Ym1 expression in macrophages by a STAT6-dependent mechanism. J Biol Chem 2002; 277: 42821-42829.

34. Odegaard JI, Ricardo-Gonzalez RR, Goforth MH, Morel CR, Subramanian V, Mukundan L et al. Macrophage-specific PPARgamma controls alternative activation and improves insulin resistance. Nature 2007; 447: 1116-1120.

35. Liu WF, Wen SH, Zhan JH, Li YS, Shen JT, Yang WJ et al. Treatment with recombinant Trichinella spiralis cathepsin B-like protein ameliorates intestinal ischemia/reperfusion injury in mice by promoting a switch from M1 to M2 macrophages. J Immunol 2015; 195: 317-328.

36. De Santa F, Totaro MG. Prosperini E, Notarbartolo S, Testa G, Natoli G. The histone H3 lysine-27 demethylase Jmjd3 links inflammation to inhibition of polycomb-mediated gene silencing. Cell 2007; 130: 1083-1094.

37. Satoh T, Totaro MG, Prosperini E, Notarbartolo S, Testa G, Natoli G. The Jmjd3-Iff4 axis regulates $\mathrm{M} 2$ macrophage polarization and host responses against helminth infection. Nat Immunol 2010; 11: 936-944.

38. Ishii M, Wen H, Corsa CA, Liu T, Coelho AL, Allen RM et al. Epigenetic regulation of the alternatively activated macrophage phenotype. Blood 2009; 114: 3244-3254.

39. Krausgruber T, Blazek K, Smallie T, Alzabin S, Lockstone H, Sahgal N et al. IRF5 promotes inflammatory macrophage polarization and TH1-TH17 responses. Nat Immunol 2011; 12: 231-238.

40. Lawrence T, Natoli G. Transcriptional regulation of macrophage polarization: enabling diversity with identity. Nat Rev Immunol 2011; 11: 750-761.

41. Tagliani E, Shi C, Nancy P, Tay CS, Pamer EG, Erlebacher A. Coordinate regulation of tissue macrophage and dendritic cell population dynamics by CSF-1. J Exp Med 2011; 208: 1901-1916.

42. Rizzo R, Melchiorri L, Stignani M, Baricordi OR. HLA-G expression is a fundamental prerequisite to pregnancy. Hum Immunol 2007; 68: 244-250.

43. Carosella ED, Moreau P, Lemaoult J, Rouas-Freiss N. HLA-G: from biology to clinical benefits. Trends Immunol 2008; 29: 125-132.

44. Munn DH, Zhou M, Attwood JT, Bondarev I, Conway SJ, Marshall B et al. Prevention of allogeneic fetal rejection by tryptophan catabolism. Science 1998; 281: 1191-1193.

45. Torricelli M, Voltolini C, Bloise E, Biliotti G, Giovannelli A, De Bonis M et al. Urocortin increases IL-4 and IL-10 secretion and reverses LPS-induced TNF-alpha release from human trophoblast primary cells. Am J Reprod Immunol 2009; 62: 224-231.

46. Guo PF, Du MR, Wu HX, Lin Y, Jin LP, Li DJ. Thymic stromal lymphopoietin from trophoblasts induces dendritic cell-mediated regulatory TH2 bias in the decidua during early gestation in humans. Blood 2010; 116: 2061-2069.

47. Du MR, Guo PF, Piao HL, Wang SC, Sun C, Jin LP et al. Embryonic trophoblasts induce decidual regulatory $T$ cell differentiation and maternal-fetal tolerance through thymic stromal lymphopoietin instructing dendritic cells. J Immunol 2014; 192: 1502-1511.

48. Tang B, Guller S, Gurpide E. Mechanisms of human endometrial stromal cell decidualization Ann NY Acad Sci 1994; 734: 19-25.

49. Hanada R, Hanada T, Sigl V, Schramek D, Penninger JM. RANKL/RANK-beyond bones. J Mol Med (Berl) 2011; 89: 647-656.

50. Theill LE, Boyle WJ, Penninger JM. RANK-L and RANK: T cells, bone loss, and mammalian evolution. Annu Rev Immunol 2002; 20: 795-823.

51. Schramek D, Leibbrandt A, Sigl V, Kenner L, Pospisilik JA, Lee HJ et al. Osteoclast differentiation factor RANKL controls development of progestin-driven mammary cancer. Nature 2010; 468: 98-102

52. Hanada R, Leibbrandt A, Hanada T, Kitaoka S, Furuyashiki T, Fujihara $\mathrm{H}$ et al. Centra control of fever and female body temperature by RANKL/RANK. Nature 2009; 462: 505-509.

53. Hutter S, Heublein S, Knabl J, Andergassen U, Vrekoussis T, Makrigiannakis A et al. Macrophages: are they involved in endometriosis, abortion and preeclampsia and how? $J$ Nippon Med Sch 2013; 80: 97-103.

Cell Death and Disease is an open-access journal published by Nature Publishing Group. This work is licensed under a Creative Commons Attribution 4.0 International License. The images or other third party material in this article are included in the article's Creative Commons license, unless indicated otherwise in the credit line; if the material is not included under the Creative Commons license, users will need to obtain permission from the license holder to reproduce the material. To view a copy of this license, visit http://creativecommons.org/licenses/by/4.0/

(C) The Author(s) 2017 